\title{
Machine learning for the educational sciences
}

\author{
Sven Hilbert $^{1}$ (1) | Stefan Coors ${ }^{2}$ | Elisabeth Kraus ${ }^{1}$ \\ Bernd Bischl $^{2}$ | Alfred Lindl ${ }^{1}$ | Mario Frei ${ }^{1}$ | Johannes Wild ${ }^{3}$ | \\ Stefan Krauss $^{4}$ | David Goretzko ${ }^{5}$ | Clemens Stachl ${ }^{6}$
}

${ }^{1}$ Department of Human Sciences, University of Regensburg, Regensburg, Germany

${ }^{2}$ Department of Statistics, LudwigMaximilians-University, München, Germany

${ }^{3}$ Department of Teaching and Learning German Language and Literature,

Regensburg, Germany

${ }^{4}$ Department of Mathematics, University of Regensburg, Regensburg, Germany

${ }^{5}$ Department of Psychology, Psychological Methods and Assessment, LudwigMaximilians-University, München, Germany

${ }^{6}$ Institute of Behavioral Science and Technology, University of St Gallen, St Gallen, Switzerland

\section{Correspondence}

Clemens Stachl, Institute of Behavioral Science and Technology, University of St Gallen, Torstrasse 25, 9000 St Gallen, Switzerland.

Email: clemens.stachl@unisg.ch

\section{Funding information}

This work has been partially supported by a National Science Foundation (NSF) Award under Grant No. SES-1758835. Also, this work has been partially supported by the German Federal Ministry of Education and Research (BMBF) under Grant No. 01IS18036A. The authors of this work take full responsibilities for its content.

\begin{abstract}
Machine learning (ML) provides a powerful framework for the analysis of high-dimensional datasets by modelling complex relationships, often encountered in modern data with many variables, cases and potentially nonlinear effects. The impact of ML methods on research and practical applications in the educational sciences is still limited, but continuously grows, as larger and more complex datasets become available through massive open online courses (MOOCs) and large-scale investigations. The educational sciences are at a crucial pivot point, because of the anticipated impact ML methods hold for the field. To provide educational researchers with an elaborate introduction to the topic, we provide an instructional summary of the opportunities and challenges of ML for the educational sciences, show how a look at related disciplines can help learning from their experiences, and argue for a philosophical shift in model evaluation. We demonstrate how the overall quality of data analysis in educational research can benefit from these methods and show how ML can play a decisive role in the validation of empirical models. Specifically, we (1) provide an overview of the types of data suitable for $M L$ and (2) give practical advice for the application of $\mathrm{ML}$ methods. In each section, we provide analytical examples and reproducible $\mathrm{R}$ code. Also, we provide an
\end{abstract}

This is an open access article under the terms of the Creative Commons Attribution License, which permits use, distribution and reproduction in any medium, provided the original work is properly cited.

(c) 2021 The Authors. Review of Education published by John Wiley \& Sons Ltd on behalf of British Educational Research Association. 
extensive Appendix on ML-based applications for education. This instructional summary will help educational scientists and practitioners to prepare for the promises and threats that come with the shift towards digitisation and large-scale assessment in education.

\section{KE Y WORDS}

Big Data, education, machine learning

\section{Context and implications}

\section{Rationale for this study}

In 2020, the worldwide SARS-COV-2 pandemic forced the educational sciences to perform a rapid paradigm shift with classrooms going online around the world-a hardly novel but now strongly catalysed development. In the context of data-driven education, this paper demonstrates that the widespread adoption of machine learning techniques is central for the educational sciences and shows how these methods will become crucial tools in the collection and analysis of data and in concrete educational applications. Helping to leverage the opportunities and to avoid the common pitfalls of machine learning, this paper provides educators with the theoretical, conceptual and practical essentials.

\section{Why the new findings matter}

The process of teaching and learning is complex, multifaceted and dynamic. This paper contributes a seminal resource to highlight the digitisation of the educational sciences by demonstrating how new machine learning methods can be effectively and reliably used in research, education and practical application.

\section{Implications for educational researchers and policy makers}

The progressing digitisation of societies around the globe and the impact of the SARSCOV-2 pandemic have highlighted the vulnerabilities and shortcomings of educational systems. These developments have shown the necessity to provide effective educational processes that can support sometimes overwhelmed teachers to digitally impart knowledge on the plan of many governments and policy makers. Educational scientists, corporate partners and stakeholders can make use of machine learning techniques to develop advanced, scalable educational processes that account for individual needs of learners and that can complement and support existing learning infrastructure. The proper use of machine learning methods can contribute essential applications to the educational sciences, such as (semi-)automated assessments, algorithmic-grading, personalised feedback and adaptive learning approaches. However, these promises are strongly tied to an at least basic understanding of the concepts of machine learning and a degree of data literacy, which has to become the standard in education and the educational sciences. Demonstrating both the promises and the challenges that are inherent to the collection and the analysis of large educational data with machine learning, this paper covers the essential topics that their application requires and provides easy-to-follow resources and code to facilitate the process of adoption. 


\section{INTRODUCTION}

Like teaching children how to distinguish cats from dogs or apples from pears, computers are increasingly able to learn patterns and rules by example. The idea that, instead of manually coding computers to perform certain tasks, we could let computers learn themselves, has a long history (Samuel, 1959). This so-called machine learning (ML) is used to automatically detect rules and patterns in data and to apply them on new data of the same kind (Domingos, 2012). Figure 1 shows the Cross-Industry Standard Process (CRISP) for ML (Shearer, 2000). As we outline in the present review, this computational approach to learning is predestined for widespread use in the educational sciences and prone to become an important analytical tool (as it already has in many other fields).

The success of educational systems is of utmost relevance for societies (e.g., Hanushek \& Woessmann, 2010, 2012). Therefore, educational research is particularly engaged in identifying and evaluating important predictors of educational achievement (see Hellas et al., 2018, for a review). In this quest, ML is likely to become a crucial factor. It is a datadriven approach for computational learning and provides a powerful novel framework for data analysis.

The manifold use-cases of ML in education are self-evident in some areas, such as intelligent tutoring systems (see Conati et al., 2018) or the prediction of student performance (Đambić et al., 2016), and understated in others, like the validation of latent statistical models (Bleidorn \& Hopwood, 2019). More than just providing new analytical techniques, ML can help educational researchers change the modelling culture towards a more reliable science with a stronger focus on actual prediction of novel data, as has been proposed by Yarkoni and Westfall (2017) for the field of psychology, but not yet been fully incorporated by the educational sciences. In particular, the focus on robust models with reliable predictions is what should be used to advance the educational science. Hence, we dedicate a section to the merit of a new philosophy for building models in educational research. Due to the high practical impact that applied ML algorithms can have on people's lives, algorithmic fairness has become an important aspect of model application to avoid issues of systematic discrimination (Chouldechova \& Roth, 2018), privacy, and sensitive data in ML (see Ji et al., 2014). Educational sciences is a discipline that often investigates latent phenomena (Kunter, Klusmann, et al., 2013; Lindl et al., 2020; Marsh et al., 2012), so the contribution of $\mathrm{ML}$ in construct validation is essential to this field. Three recent reviews on $\mathrm{ML}$ in personality assessment have been concerned with this challenge (Bleidorn \& Hopwood, 2019; Stachl, Pargent, et al., 2020; Tay et al., 2020) and provide valuable approaches for the educational sciences. Also, in the present overview we extend beyond the topic of mere data mining in education, which has triggered several interesting reviews already (e.g., Ali, 2013; Guleria \& Sood, 2014; Romero \& Ventura, 2020).

Often, two types of ML are distinguished: supervised and unsupervised learning. The latter subsumes clustering and other exploratory data analysis techniques where no target variable (in the classical context typically called 'dependent variable') is available. In this article, we focus on supervised $\mathrm{ML}$, which models an input-output relation (i.e., the relation between covariates and a target variable).

Obviously, fitting predictive models to data is nothing new. Nearly all researchers in educational sciences are aware of the classical framework of linear regression and its associated statistical inference. Inference statistics and supervised ML both deal with the same problem of learning predictive models from data and are based on the same mathematical principles. In a sense, supervised $M L$ is predictive statistical modelling with a strong focus on non-parametric models. Here we focus on this predictive aspect of $\mathrm{ML}$ and therefore refer to supervised predictive learning when discussing ML approaches. 


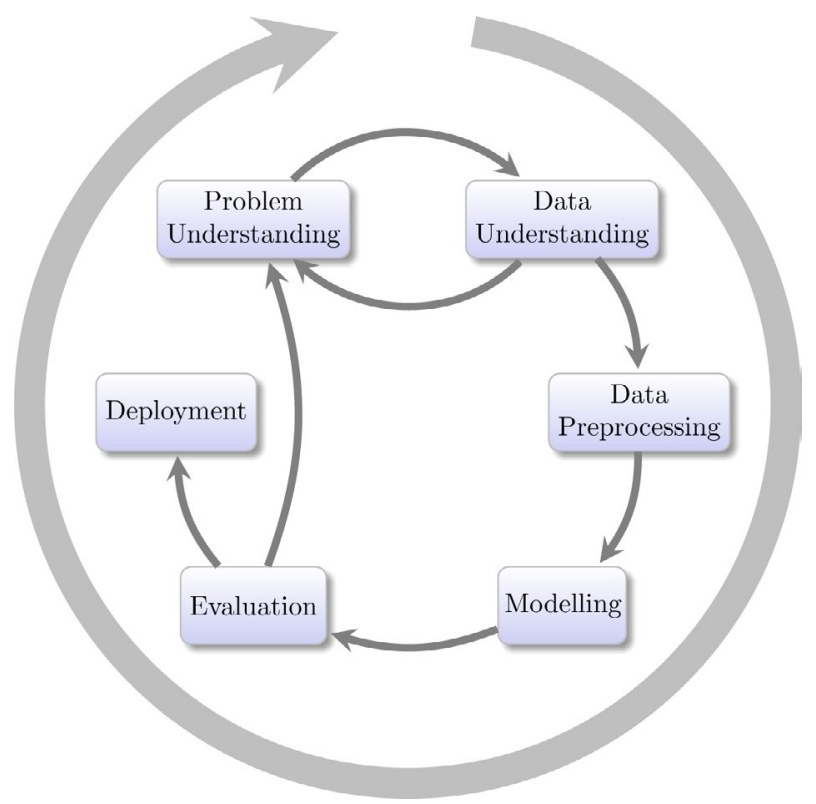

FIG U RE 1 CRISP-DM: The cross-industry standard process for data mining, which also applies to machine learning. The circular process starts with the problem definition (Appendix S2) and the data situation (Section 2). The collected data (Section 2.2) is then used for modeling (Section 3) following data preprocessing (Section 3.4). The resulting model is evaluated (Sections 3.2 and 3.6) and deployed if successful. Alternatively, the process starts again

But, as we explain in the section 'Analysis', the two fields differ in subtle ways, often further complicated by different cultural and philosophical approaches to the same underlying empirical problems, in addition to frequently using different terminology for the same concepts. A major historical difference has always been ML's focus on strong predictors that generalise well beyond the training data, in order to use them in automated environments. Classical statistics traditionally focused on explaining relationships in data for the sake of gaining scientific insights and quantifying the associated uncertainty. After computational statistics' renewed interest in non-parametric modelling and the recent arrival of interpretable machine learning (IML), the lines between the fields have become even blurrier.

In this instructional summary, we show how the rise of ML techniques strongly affects education in general and how this development puts the educational sciences in a crucial position: the incorporation of these new methods is warranted on two main levels, namely (1) the collection of data, and (2) the analysis of data. Additionally, we provide an overview of practical ML applications for education in Appendix S2. We combine elements from a review (e.g., past findings) with the practical exercises and examples of a tutorial paper, and with the critical positions and reflections of a perspective piece (e.g., algorithmic fairness). Following this multifaceted approach, this format allows educational scientists to profit from embracing $\mathrm{ML}$ and learn from the experiences of other scientific disciplines that have already implemented $M L$ into their standard repertoire. Moreover, ML provides the opportunity to at least extenuate many problems of current educational research (e.g., a large amount of non-replicable results, as discussed in the section 'Prediction and explanation').

Naturally, predictive modelling has become more prominent in the educational sciences since large digital datasets have become available. These (mostly new) forms of data collection and assessment have to be contextualised before the potential of ML for the educational sciences can be fully understood. Hence, the first part of this article focuses on novel methods for data collection and assessment suitable for ML models. We particularly consider 
the cases of Big Data in education (see Hadioui et al., 2017; Teasley, 2019) and focus on large-scale studies (e.g., Depren et al., 2017; Yoo, 2018). In the second part, we focus on ML modelling. Specifically, we outline the common procedures involved in computational modelling and highlight typical pitfalls and caveats relevant to the educational sciences. To help educational scientists deal with these analytical issues, a discussion of prediction-centred model evaluation, interpretability, replicability and model validation is provided. Additionally, we provide theoretical and practical examples, give concrete advice and indications for the analysis of educational data with $\mathrm{ML}$, and offer R-code, using the m/r3 package (Lang et al., 2019), to accompany each section on https://osf.io/ntre9/ as well as a discussion of MLbased practical applications that can be used as educational tools in Appendix S2.

\section{EDUCATIONAL (BIG) DATA}

Today, enormous amounts of different data are continuously generated and collected from various types of sources, such as people, machines or sensors. These data hold information on behaviour, expressions or context. Their collection oftentimes does not follow a specific research question but is collected due to the opportunity or to provide a database for possible future studies (Markowetz et al., 2014). This special phenomenon of our 'digital age' is often described by the term Big Data, which has been omnipresent in everyday life and in research for about a decade. However, as past work has shown (e.g., Baro et al., 2015; De Mauro et al., 2016; Favaretto et al., 2020; Ylijoki \& Porras, 2016), an exact definition of this term has yet to be achieved-perhaps due to the ongoing changes and the dynamics of the associated areas of life and research. Different approaches start with the phenomenon itself, its features or the (analytical) methods evoked by it, such as ML (Hellas et al., 2018; Yarkoni \& Westfall, 2017), and include different aspects, depending on the focus of consideration (e.g., Baro et al., 2015; De Mauro et al., 2016; Ward \& Barker, 2013; Ylijoki \& Porras, 2016). Common to more than $70 \%$ of the definitions examined by Ylijoki and Porras are the three central criteria—volume, variety and velocity (cf. De Mauro, Greco \& Grimaldi; Favaretto et al., 2020; Laney, 2001; Ward \& Barker, 2013). Additional facets, such as value, veracity, complexity or impact are added in less than $30 \%$ of the descriptions (Baro et al., 2015; Ylijoki \& Porras). Volume refers to the almost immeasurable amount of data that gives Big Data its name, variety to the multitude of data, and data types that differ greatly and have little or no structure. Velocity describes two aspects of Big Data, namely the enormous rate at which the data is generated and on the speed at which the rapidly growing amount of data is processed-often within (fractions of) seconds-in order to be able to react to it as quickly as possible.

\section{Voluminous data}

Large amounts of data, usually perfectly suited for the application of ML algorithms, are regularly created in educational research, as can be seen directly from an exemplary consideration of central topics. The following sections discuss the prediction of academic performance for individuals and in the context of national and international studies on educational monitoring. In addition, we summarise data on research regarding teaching quality, teacher competencies and academic performance.

As Hellas et al. (2018) point out in their review of more than 350 publications, research on predicting academic performance regularly generates a vast amount of data due to the large number of variables that can be used to measure academic performance (e.g., GPA, individual grades or programme retention) and the even larger number of factors that are related 
to it (e.g., demographics, personality, academic, behavioural or institutional dimensions). The generated data are analysed with a continuum of methods, that 'ranges from basic statistical analysis and correlations to the use of state of the art machine learning methods' (Hellas et al., 2018, p. 185). These studies are comprehensive, but mostly include regionally limited investigations. Hence, for international comparisons, extensive data are collected from students for each implementation. This is especially true for subjects like mathematics and the natural sciences, as displayed by, for example, the international TIMSS (Trends in International Mathematics and Science Study), in which thousands of variables about mathematics classes and students were collected.

Since 1995, TIMSS has been measuring the performance of more than 300,000 students in mathematics and science at the end of the fourth grade in over 50 participating countries and regions every four years. The collected data include information on the respective educational systems; individual, family, and socioeconomic backgrounds; on attitudes towards mathematics; on characteristics of teachers, classes and schools; on instructional design; and curricular aspects (e.g., Mullis et al., 2016). Since 2001, Progress in International Reading Literacy Study (PIRLS) has evaluated and internationally compared the reading comprehension of students at the end of the fourth grade every five years. Similar covariates were used to identify favourable and unfavourable factors for the development of reading comprehension (e.g., Mullis et al., 2017). However, the most comprehensive international large-scale study, carried out every three years since 2000, is the Program for International Student Assessment (PISA), which measures the performance of more than 600,000 15-year-olds from 79 countries in reading as well as mathematical and scientific literacy. The tests, which are mainly computer-based, are supplemented by electronic questionnaires for the students, their parents, teachers and school principals. This way, information on the socioeconomic status of the family, the migration background, learning contexts in the respective class or school, learning habits and strategies, (out-of-school) media use, and students' attitudes and motivations are collected (e.g., OECD, 2019a, 2019b, 2019c, 2020a). Thus, not only do several hundred thousand students annually participate in these studies, but the resulting data also contain thousands of variables for each participant.

In addition to the investigation of student-related factors of competence, extensive studies on the quality dimensions of teaching are also flourishing in current educational research (see Praetorius et al., 2018, for an overview). The evaluation and assessment of teaching qualifications is carried out primarily from the students' perspective (some alternatives are outlined below) and based on often extensive catalogues of criteria that are founded on various theoretical approaches (e.g., Lipowsky et al., 2009; Praetorius et al., 2018; Wisniewski et al., 2020). To empirically underline the structural and predictive validity of the postulated models, samples of thousands of students are acquired with specific paper-based questionnaires (e.g., Kunter, Baumert, et al., 2013), but also by means of digital applications for the evaluation of their teachers' teaching (Wisniewski et al., 2020).

Teachers are central factors in the process of learning (Hattie, 2009). Several large-scale studies have investigated the professional competence of teachers and its development in various teacher-education systems across nations (e.g., Mathematics Teaching in the 21st Century [MT21], Teacher Education and Development: Learning to Teach Mathematics [TEDS-M], Quality of Instruction in Physics [QuIP], Teaching and Learning International Survey [TALIS]). Following the core components of professional competence (Shulman, 1986), the focus of these studies lies on the evaluation of professional knowledge (content knowledge, content knowledge and pedagogical knowledge) beliefs, values, goals, motivational orientation and self-regulation (e.g., Kunter, Baumert, et al., 2013). These latent constructs were examined using large samples of student teachers, trainee teachers and working teachers in up to 48 different countries (Fischer et al., 2014; Ingvarson et al., 2013; OECD, 2019d, 2020b; Schmidt et al., 2011). The constructs were initially assessed with 
newly constructed paper and pencil tests and questionnaires but several of these tests have since been digitised (e.g., Kaiser et al., 2017; Kersting et al., 2012; see also the section 'Velocity of data'). Other studies went one step further and investigated the interrelationships between teachers' professional competence, instructional quality, students' cognitive performances and their affective states. By combining the theoretical and methodological approaches of the three research fields, the amount of data produced in such research projects (e.g., Campbell et al., 2014; Hill et al., 2005; Kelcey et al., 2019; Kunter, Baumert, et al., 2013) is continuously growing and proved to be extensive, multi-layered and complex. In addition, contextual characteristics of classes (e.g., schools or country-specific education systems) can be a challenging aspect of the analysis and variable selection often seems arbitrary or based on narrow theoretical aspects.

\section{Variety of data}

Apart from the availability of large-scale datasets, the change in modalities of how data is collected and the increased use of new types of data are additional reasons for the educational sciences to start embracing ML methods (Hellas et al., 2018). To fully understand the potential of $\mathrm{ML}$ in education, it is important to understand the various types of data (e.g., text, video and sensor data) that can be used in the process (Romero \& Ventura, 2010). In addition to traditional paper and pencil questionnaires and tests, digitised versions of these instruments are used to investigate various aspects of teachers' competence. Sometimes, digital reaction time tests (e.g., Kunter, Baumert, et al., 2013) and criteria-based video vignettes (e.g., Bruckmaier et al., 2016; Kaiser et al., 2017) are used for assessment. The systematic categorisation and the analyses of teaching materials by trained coders are also part of the common repertoire of methods in the learning sciences (Kunter, Baumert, et al., 2013). Formerly lab-based, complex eye-tracking procedures are increasingly deployed via mobile devices in naturalistic classroom settings (see Beach \& McConnel, 2019, for a review). These methods are nowadays used to assess the quality of teaching (see Fauth et al., 2020; Praetorius et al., 2018, for an overview).

Moreover, the accelerating transition of educational activity to online courses and computer-aided tutorials, often subsumed under Education 4.0 (see Ciolacu et al., 2017), increases the amount of available digital data. Fine-grained log-files from student-computer interaction can be used to extract complex, real-time information from online testing (e.g., Hellas et al., 2018; Juhaňák et al., 2019) or learning management systems (see Ciolacu et al., 2017). More indirect measures, such as digital footprints (see Kosinski et al., 2015; Lambiotte \& Kosinski, 2014) or even high-frequent mobile sensing data (Harari, Müller, et al., 2017), are prone to become more prevalent in the educational sciences and are resulting in an enormous quantity and variety of-often strongly interrelated-variables. This largescale character makes it impossible to include all available information in classical linear models.

Mobile sensing refers to the use of mobile, sensor-equipped, technical devices to register, store and process (Eagle \& Pentland, 2006; Lane et al., 2010) data on the users' behaviour (Harari, Müller, et al., 2017) and context or whereabouts (Harari et al., 2018). In contrast to classical questionnaire data in a digital context, such as experience-sampling (see Reis \& Gable, 2000), mobile sensing does not require 'querying' participants to report their behaviour themselves but registers actions as they unfold on a device or in close proximity to it (i.e., passive sensing). In psychological research, mobile sensing has been used to investigate everyday behaviour (e.g., social and communication behaviour, mobility, physical activity, media use and day-nighttime activity) and to relate these behavioural metrics to individual differences in personality (Budimir et al., 2020; Harari et al., 2019; Montag et al., 
2015; Schoedel et al., 2018, 2020; Stachl, Au, et al., 2020; Stachl et al., 2017). Further, in the health sciences, behavioural data from smartphones has been reliably related to psychological well-being (Cornet \& Holden, 2018; Servia-Rodríguez et al., 2017) and to the severity of clinical depression (Saeb et al., 2016). Although mobile sensing has become popular in multiple fields, very little research on this topic exists in the educational sciences (Barkley \& Lepp, 2016; Harari, Gosling, et al., 2017). Yet, for many research topics, such as the prediction of retention by online activity or the monitoring of digital learning strategies, the use of mobile sensors could be promising.

Additionally, the automated collection and analysis of large amounts of data for the prediction of outcomes has become feasible due to advances in computation technologies and the widespread use of digital devices for education (Jarke \& Breiter, 2019). The potential of this approach is enormous: for a comparison, Kosinski et al. (2015) published the myPersonality Facebook application, which was widely regarded as a milestone of digital assessment in personality psychology and included personality profiles from close to 7.5 million Facebook users with about 2 million of them also sharing data on their Facebook profiles. Notably, this is far less than a standardised digital survey in education could achieve if standardised tests, such as the Scholastic Assessment Test (SAT; over 2 million students per year), were analysed. At this moment, the educational sciences have the advantage that they can build upon the experiences and developments from other fields, such as psychology, the health sciences and various others, to use its key resources for the economic collection of valuable educational data. But dealing with these new and different types of data requires intensive preprocessing (see the section 'Preprocessing'), adequate models, theoretical expertise and knowledge on analytical pitfalls.

\section{Velocity of data}

In the educational sciences, complex, often latent, target constructs and performance characteristics, such as cognitive, social and emotional competencies of students and teachers, are measured. According to contemporary definitions and conceptualisations (e.g., Blömeke et al., 2015; Kunter, Baumert, et al., 2013; Weinert, 2001), these constructs manifest in specific situations through knowledge-based behaviour. Moreover, they are only partially consciously reflectable, mostly non-verbalisable, and thus only partially measurable through classical paper-based test or questionnaire stimuli (Heine \& Reiss, 2019). Although digitised versions of these instruments (e.g., with additional video elements; Bruckmaier et al., 2016; Kaiser et al., 2017; Knievel et al., 2015) offer context-rich possibilities for capturing these behaviours in real-time, these ultimately just replace textual vignettes with videographic ones, whose greater veracity is subject to critical discussion (e.g., Kaiser et al., 2015; Knievel et al., 2015).

Regarding these constructs, digital survey formats only add value if (as is now the case for PISA) interactive elements are included in the tasks. These can only be meaningfully realised in computer-based testing, such as real-time simulation scenarios in natural sciences or innovative item formats (OECD, 2019a, 2019b, 2019c, 2020a). Computer-based testing will not only improve the veracity and the content validity with regard to the measured constructs and make data collection more structured, timesaving and thus more efficient, but also more variable and dynamic. Moreover, a multi-stage adaptive test design can increase the measurement accuracy if, for example, the selection and order of test items is controlled by an adaptive ML-algorithm that considers the responses of the preceding items. Furthermore, additional meta and/or process data can be collected that go beyond mere processing times for sub-tasks or stopping points in videos. This includes log-files in which every click, every movement, and every position of the cursor or-more generally-every (predefined) user 
action can be registered and nearly at the same time related to the presented problems. For this data, which are generated rapidly and thus consist of various sources and types with different scales and diffuse encodings (Ciolacu et al., 2017), ML methods can be used to validate latent constructs, related to such actions (Bleidorn \& Hopwood, 2019).

Numerous other approaches that are regularly used in combination with $\mathrm{ML}$ analysis are hardly applied in the educational sciences. Examples are high-frequent behavioural data from mobile sensors (as discussed above), intelligent tutoring systems and computer-supported collaborative learning, individual online learning environments (including computer-adaptive testing), and massive open online courses (MOOCs) with thousands of students (e.g., Baker \& Yacef, 2009; Kennedy, 2014). These methods can quickly provide the necessary data to identify critical situations in individual educational trajectories or learning difficulties. They can also be used to predict student- performance and learning outcomes and promptly suggest personalised support and interventions (e.g., through automated recommender systems). Moreover, the often time-consuming and cost-intensive preparation of comprehensive, complex and continuous or fine-grained data sources can be supported and facilitated by ML methods. Examples of this are automated processing, classification and evaluation of responses to test items, written essays, audio recordings or even (instructional) videos in the context of natural language processing (see Appendix S2 for opportunities and limitations).

\section{ANALYSIS}

The goal of any supervised ML model is to learn the functional relationship between the input variables (i.e., the covariates or features, as they are called in the ML context) and the output variable(s) (i.e., the target variable). In educational research, the input variables are often quite heterogeneous and range from demographic data to psychometric factors, such as self-regulation or self-efficacy, whereas the target variables are in many cases a measure of academic performance (Hellas et al., 2018). The model learns this relationship based on empirical data for which we assume that both feature and target values have been observed. Target values are called labels in ML. The data are the collected measurements for our objects of interest, such as the test scores of students. We usually assume that our data have been sampled from an underlying, unknown probability distribution, often called the data-generating process, in an identical and independent (i.i.d.) fashion. In supervised learning, two common prediction tasks exist, which simply depend on the type of the target variable: classification, with a categorical variable (binary- or multiclass classification), and regression, with a numerical variable. Although regression tasks are the most widely applied analytical approach in the educational sciences (Hellas et al., 2018), classification techniques are also common, such as for the identification of students at risk (Mashiloane \& Mchunu, 2013).

We commonly have two different goals in learning this functional relationship: learn to predict emphasises that we want to use our obtained model on new data (e.g., new students) where the target label is unknown (historically, this is the focus of $\mathrm{ML}$ ), whereas learn to explain emphasises a motivation to understand and study the modelled relationship between the input and output variables to foster the understanding of the functional relationship and potential theoretical implications, and to generate new hypotheses for confirmatory research. In many applications, both goals are of interest, yet to different degrees. Supervised learning can be described by three, mainly orthogonal components (Domingos, 2012):

1. A set of parameterised functions that we select from, during the model fitting process. In $\mathrm{ML}$, this is called the hypothesis space. An example would be all possible linear models with two parameters $\beta_{0}$ and $\beta_{1}$, representing intercept and slope, respectively. 
In this case, the hypothesis space includes any linear relationship between the input variable and the output variable.

2. A function that assigns each possible model to a numerical score that expresses how well our model fits our data. In ML, this is typically called empirical risk and is minimised to find the optimal model; in statistics, this score is typically called the likelihood of the model and is maximised. The empirical risk is defined as a measure of the total deviations between predictions and true labels-for each observation, we measure how much the prediction differs from the true label. This pointwise measure of difference is called a loss function in ML. An example for such a function would be the sum of the squared differences between the predictions and the labels in a regression task.

3. An optimiser that systematically searches for the best parameterisation of our model space by optimising its numerical score with respect to the dataset at hand. Gradient descent, for example, is an optimiser that searches for the minimum of the empirical risk function in stepwise fashion by calculating its gradient at a point and selecting the next point in the opposite direction of the gradient.

In short, we need (1) a set of models to choose from, (2) a value that tells us how well the model fits the data, and (3) a function that tells us how to find the best-performing model. Through this lens, predictive modelling (or supervised $\mathrm{ML}$ ) is reduced to mere parameter optimisation or curve fitting. Notably, although we described the above principles from a ML point of view, the same general procedure applies classical statistical model fitting. Also, risk minimisation in $\mathrm{ML}$ and statistical likelihood maximisation are mathematically equivalent. Therefore, the general principle of predictive modelling is easily relatable to processes already known to educational researchers familiar with linear regressions and more general statistical models (see Theobald et al., 2019, for a review of typical quantitative methods in education).

This section treats the individual parts of the learning process, starting with a short introduction to different types of models and subsequently discussing the resampling and the tuning processes, which are used for the evaluation and the optimisation of models, respectively, and highlights the connections and applicability to educational research. An additional section is dedicated to the preprocessing of data. The subsequent parts discuss the interpretation and philosophical consequences of this approach for educational research and highlight the challenges that come with it.

\section{Model classes}

Because the available range of predictive models is extremely large (see Appendix S1), we are not able to review the entire range of approaches but refer to Friedman et al. (2001) for a comprehensive textbook. Note that the information and properties provided in Appendix S1 refer to the original algorithms and their most common implementations. A systematic overview of model classes used for the prediction of academic performance is provided by Hellas et al. (2018).

Linear models are simple regression models that transform the feature vector into an output score through a linear function. For the logistic regression, which addresses binary classification problems, this score is further transformed through the logistic function into output probabilities (i.e., a value between 0 and 1). The concept of further transforming the score obtained by the linear function is called generalised regression in statistics. So, while features are combined in a linear fashion to a single score through the subsequent transformation, they act in a non-linear fashion on the modelled probabilities. 
Because linear models can be applied with limited sample sizes and generalised to more complex relationships, linear regressions are among the most common analytical techniques in educational research (Theobald et al., 2019).

Regularised linear and logistic regression models add a (weighted) penalty to their risk function, which penalises 'more complex models'. Here, 'complexity' is usually measured by the number of parameters that are far away from zero, as a zero-coefficient would completely switch off the effect of its associated feature. Regularisation prevents overfitting and makes parameter estimation in $p>n$ conditions feasible.

The weight of this penalty term can be tuned, in a data-driven manner, so that the complexity of the model is selected appropriately for the task at hand. The most common regularised regression models are the LASSO (least absolute shrinkage and selection operator) regression (Tibshirani, 1996) that can shrink parameter estimates to zero (integrated variable selection), the ridge regression (Hoerl \& Kennard, 1970), and a combination of both approaches - the elastic net (Zou \& Hastie, 2005). As in (generalised) linear regression, the linear form of the model specifies how the features and the target variable are connected. In education, this is often feasible to predict dichotomous variables, such as dropout, and regularised logistic regressions can be highly effective in such tasks (Aulck et al., 2016). It is often necessary to explicitly transform the input variables to account for non-linear relationships-or to switch to a genuinely non-linear model.

Kernel-based methods can be an alternative for these cases. They apply a so-called kernel-function to the predictor variables, which implicitly lifts the feature space to a higher dimension (for an introduction to kernel-based ML, see Schölkopf \& Smola, 2003). The most common instance is the support vector machine (Cortes \& Vapnik, 1995), which is mainly used for classification tasks, in which it determines classification boundaries in high-dimensional spaces, such as for the prediction of academic performance categories (Burman \& Som, 2019) or dropout (Kesumawati \& Utari, 2018), but can also be applied to regression problems (Drucker et al., 1997).

A fourth class of models is based on decision trees (Breiman et al., 2017). Decision trees recursively apply binary splits to the input space (or training data) to obtain regions of input space with minimal variance of the outcome (the so-called 'leaves'), as trees model the outcome in the resulting leaf regions by a simple constant (i.e., for all data points in one leaf, the same outcome is predicted). This recursive structure allows for interactions among predictor variables and non-linear modelling.

The splitting is usually early-stopped, or the tree is pruned post-hoc (i.e., cut down to a smaller size without losing much prediction performance) to prevent overfitting. Single decision trees typically lack predictive power, so ensembles of trees are used instead, usually in the form of a random forest (Breiman, 2001a, 2001b) or gradient-boosted trees.

The most common tree-based method, the random forest, estimates an ensemble of different trees. It uses bootstrap aggregation (bagging) by Breiman (1996) to inject randomness by fitting multiple trees trained on different bootstrap samples of the training data. An additional split-variable randomisation also constrains the searched features for each split to a randomly selected subset. Together with bagging, this leads to less correlated trees within the ensemble. The predictions of those individual trees are then averaged to obtain a final prediction (majority vote for classification). While general bagging can be combined with any learning algorithm, tree-bagging as in random forests has proven to deliver state-of-the-art performance, so that they are among the most popular ML methods in predicting academic performance (Hellas et al., 2018). In a random forest, each tree is typically maximally extended up to only a few observations per leaf to obtain minimal correlation between each tree. Another important aspect of random forests for educational research is that they have been shown to be readily combinable with psychometric models, such as the Rasch model (Golino \& Gomes, 2016). 
Neural networks are another popular class of learning methods that can be applied to classification as well as regression tasks. The idea of neural networks is to combine multiple, simple computational units or simple functions (neurons) in a complex graph-like network, usually by ordering the neurons into multiple, sequential layers. Each neuron usually performs a weighted linear combination of its inputs together with a univariate non-linear transformation to compute its output. The layers of the network now can be interpreted as vector-wise transformations of the input-vector into different vector-form representations, through an application of sequential affine-linear (i.e., without a fixed origin) and componentwise non-linear transformations, until the output representation is reached.

Neural networks have currently shown their best results on tensor data, such as images, videos and sound, and due to their flexible architecture are certainly one of the most versatile ML techniques. On the other hand, for the common case of tabular data, random forests often either show equal or better performance and currently demand less tweaking from the modeller. However, complex tasks in large datasets from the field of education, such as automated essay scoring and sequential data from MOOCs, are often a good fit for analyses with neural network models (Saito \& Watanobe, 2020). For a good overview of neural network models, see Aggarwal (2018).

Choosing the correct model for a problem at hand can be challenging, because even if predictive performance is the only measure of interest, the best-performing learning algorithm cannot be identified in advance.

It is therefore advisable to start the analysis with a simple baseline model like a (generalised) linear model or a decision tree. Since these models are easily and naturally interpretable, they serve as an ideal entry-point. For a simple-to-handle non-linear analysis, a random forest is recommended as a next step, as this model often achieves strong predictive performance in many applications but does not require much preprocessing of the input data or too much parameter tuning and adaptation. Only after the performances of these simpler models have been evaluated, more difficult model classes like neural networks should be considered. These models often require much more difficult tuning and are still very challenging to interpret. Performing a benchmark analysis of different learning algorithms competing against each other is a typical way in $\mathrm{ML}$ to find the most predictive model.

\section{Evaluation and overfitting}

For a classical statistical model, the goodness-of-fit is typically evaluated. For a linear regression, as explained in detail below, one can calculate the $R^{2} \in[0,1] . R^{2}$ indicates the percentage of variance the model explains regarding the data used to estimate it and is likely to be the most familiar index of model fit for educational researchers. This implies that training error can be a poor statistical estimator for generalisation performance and be severely optimistically biased. On the other hand, as already foreshadowed in Figure 6, a remarkably simple remedy exists in the form of test error estimation: the dataset is split into two parts (one for training, the other for testing) so the target variable is predicted using the trained model and the deviance from the true values is quantified. Typically, for a single data split, two thirds of the complete sample are used to train the model and one third to test its generalisability. This procedure enables an unbiased estimate of the generalisation error, as the performance is never tested on those data that are used for model building.

Differentiating between the error obtained for the training set and the generalisation error is very important. The generalisation error quantifies the expected amount of incorrectness when the model is used to predict the target in a test sample. In other words, it quantifies how well the model generalises to new data. 
For example, the dropout rate for a sample of students may be predicted perfectly by a very flexible model, but in most cases, the interesting question is how well it does in predicting the dropout rate for new groups of students. Therefore, evaluation in predictive modelling, to a large part, means studying how to construct reliable statistical estimators for the generalisation error.

A sufficiently flexible model can learn the complete pattern of the training data, which can result in overfitting, because random noise in data becomes part of what is learned. Overfitting is mainly determined by the following properties:

- It is more likely, the more flexible our model space is. Deep neural networks can overfit more easily than a simple linear regression.

- It is more likely, the more aggressively we optimise the model class. ML often uses simpler optimisation techniques and early stopping of optimisation to counter overfitting here.

- It is more likely, the more features we have at our disposal for modelling.

- It is less likely, the more data we have for training. Given an infinite amount of data, overfitting, in theory, does not happen. Unfortunately, in the educational sciences, one often must deal with rather limited sample sizes.

This implies that overfitting may also occur with linear models, such as in small sample situations where the feature space is sufficiently high-dimensional (which is why an adjusted $R^{2}$, corrected for the number of features, is often reported). This phenomenon causes the resulting model to make no or only very few prediction errors for the training data, while performing poorly with new, unknown data, in terms of predictive accuracy, which is what we are ultimately interested in.

Figure 6 shows the principle of overfitting for the example of a polynomial regression. Using a low-degree polynomial, the regression is not capable of modelling the truly underlying distribution properly due to inflexibility (underfitting). In this example, overfitting starts after using a degree of five.

Various metrics for the evaluation of both classification and regression are available. A good overview of the most common ones is given by Janocha and Czarnecki (2017) and provided in Appendix S1. In regression, the mean squared error (MSE) is frequently used to quantify model performance: the differences between all true values and respective predicted values are squared, added up and divided by the number of cases. The lower the MSE, the better the model performance. As outlined above, $R^{2}$ is a comparison of MSEs of two different models. On training data, its value lies between 0 and 1 (as a linear model is more flexible than a single constant). The ratio of the two is subtracted from 1 to simply turn it into a positive measure where $R^{2}=1$ corresponds to the optimal value (MSE of the linear model equals 0 ) and 0 corresponds to the worst possible value (the MSE of the linear model equals the MSE of the constant model). Note that this metric could also be applied to test data, in which case $R^{2}$ could also become negative, due to overfitting.

The mean absolute error (MAE) is a similar metric which uses the absolute error between all true and predicted values instead of the squared errors. In comparison to the MSE, omitting the squaring of the error renders this metric more robust against outliers in the data.

The most common performance measures used for classification tasks are the rate of wrongly classified labels, that is, the classification error (CE) and its counterpart, the accuracy $(A C C)$, which is the rate of correctly classified labels.

In cases of (highly) imbalanced data and/or where prediction errors on one class can have a more negative effect than the other, accuracy and classification error are not an ideal choice. A good hybrid is provided by receiver operating characteristic (ROC) measures, which are based on the confusion matrix in Figure 2. The confusion matrix tabulates true 
classes versus predicted classes and therefore displays the number of errors per class and prediction category.

A related approach are $\mathrm{ROC}$ curves for binary classification, often used when classes are imbalanced or of different importance, but the costs of misclassifying these classes are unknown Fawcett (2006). In ROC analysis, it is assumed that the classifier produces a numerical score (or probability) which indicates its preference for the positive class. Given a threshold, one can now dichotomise these scores, hence each threshold gives rise to a different binary classification and thus its own confusion matrix. Due to the unknown costs, it is unclear which of these thresholds is preferable. ROC analysis computes the true positive rate and false positive rate (or the true positive rate and the positive predictive value) for all potential thresholds (i.e., for all resulting confusion matrices) and visualize them as a curve (Figure 3). This approach has been found to be particularly helpful for decision making in the educational context, such as early warning systems for dropout (Bowers \& Zhou, 2019) or to improve curricular content (Campo et al., 2010). In education, the combination of specificity and sensitivity is particularly important to, for example, not miss students at risk but on the other hand not waste resources on students actually not in need (Kilgus et al., 2012). In order to compare different classification models, ROC curves can be aggregated to a single value by calculating the Area Under the Curve (AUC), which is a widely used performance measure. It can be interpreted as the probability of the model to rank a randomly chosen positive observation higher than a random negative one. This is equivalent to the Wilcoxon rank-sum test (Hanley \& McNeil, 1982).

Possible values for the AUC lie between 0 and 1 . However, for a binary classification task, a value of 0.5 can already be obtained by chance - that is, a model which randomly labels its predictions based on the prior class distribution. Consequently, a useful model should clearly outperform this value. Aulck et al. (2016), for example, use the AUC to compare the performance of a regularised logistic regression, a random forest, and a $k$-nearest neighbours algorithm and show that the logistic regression outperformed the other models in predicting student dropout.

Although the AUC is naturally limited to two-class problems, its idea can be transferred to multiclass classification in several ways. A good overview of common multiclass classification AUC measures can be found in Hand and Till (2001).

The multitude of performance measures may seem confusing at first glance and especially the choice of a suitable measure is not always clear. It largely depends on the underlying application and, of course, the associated target variable and some recommendations can be made, such as $A \cup C$ and $F_{1}$ for binary classification tasks. For multiclass problems, the Log-Loss could be used, whereas for regression, the MSE is a typical choice as performance measure.

\section{Resampling}

In order to capture and smooth out the inherent variance in ML test error estimation, resulting from the random construction of train-test splits on a given dataset, the holdout process is typically repeated and all test performances are averaged (Bischl et al., 2012) (Figure 4).

One of the most common practices to evaluate model performance in ML is called crossvalidation $(\mathrm{CV})$ - it is illustrated in Figure 5 . In order to most efficiently and uniformly use the available data, CV partitions the data into $k$ disjoint test sets, where $k$ denotes the number of iterations, the so-called folds. In each iteration, a single fold is selected for testing, while the model is trained on the remaining folds. At the end, all test-block performances are averaged. This technique is so popular because it effectively 'recycles' the sample by using each of the $k$ splits one time as test set, so that also each observation is tested exactly once. 
Truth

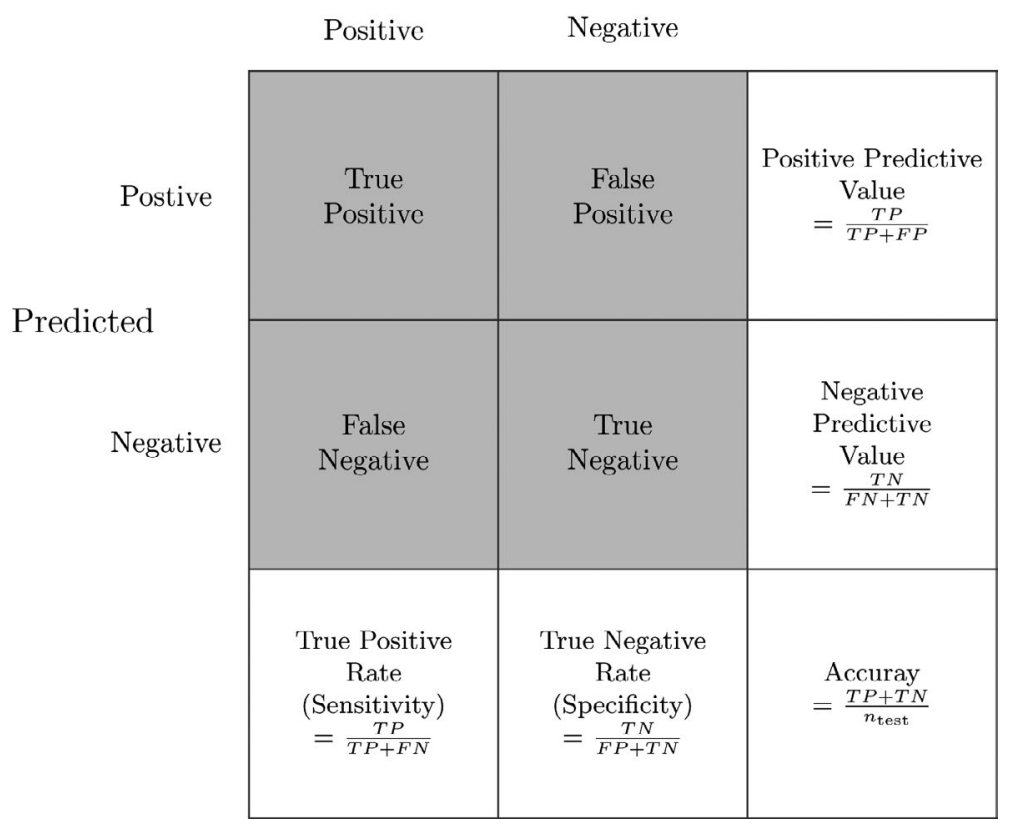

FIGURE 2 Confusion matrix

Especially on smaller datasets, many train-test repeats with smaller test set sizes should be performed. For data with a few thousand observations, 10 -fold CV is a recommended standard. Small sample size scenarios with $n<500$ or $n<200$, other techniques might be considered. Here, leave-one-out CV, where each training set of the CV consists of $n-1$ data points, and one tests on only one 'left out' example is often recommended. However, the estimator is known to have considerable variance and modern evaluation rather recommends repeating the $\mathrm{CV}$ multiple times with a high number of folds and repetitions and average over both repetitions and folds (Bischl et al., 2012).

A word of warning: even if the available dataset is large and one would therefore like to choose a simple train-test holdout approach (i.e., $k=1$ ) for computational simplicity and efficiency, small sample size effects can still be active. Typical situations unfold when classes in binary classification or observation subgroups (e.g., defined by one or multiple categorical features) are of highly imbalanced sizes and some groups of interest are rather small. In such cases, repeated CV might still be the recommend choice. An advisable rule of thumb is: if the measure of interest exhibits a high variance, smooth it out by repetition.

Another important consideration is the stratification of the sample: if the data are highly imbalanced regarding a potentially influential class variable, it is recommended to stratify during resampling, which is to keep the class ratio of the target variable constant across all splits according to the overall class ratio (e.g., the same ratio of men and women in a gender prediction task or the same ratio of different school types). Moreover, if the data contain spatial or temporal structures, it is most often desirable to keep them intact during resampling. For example, if the data is sampled from three different school years, it can be advisable to train on the data of two years and predict on the data of the remaining year. 


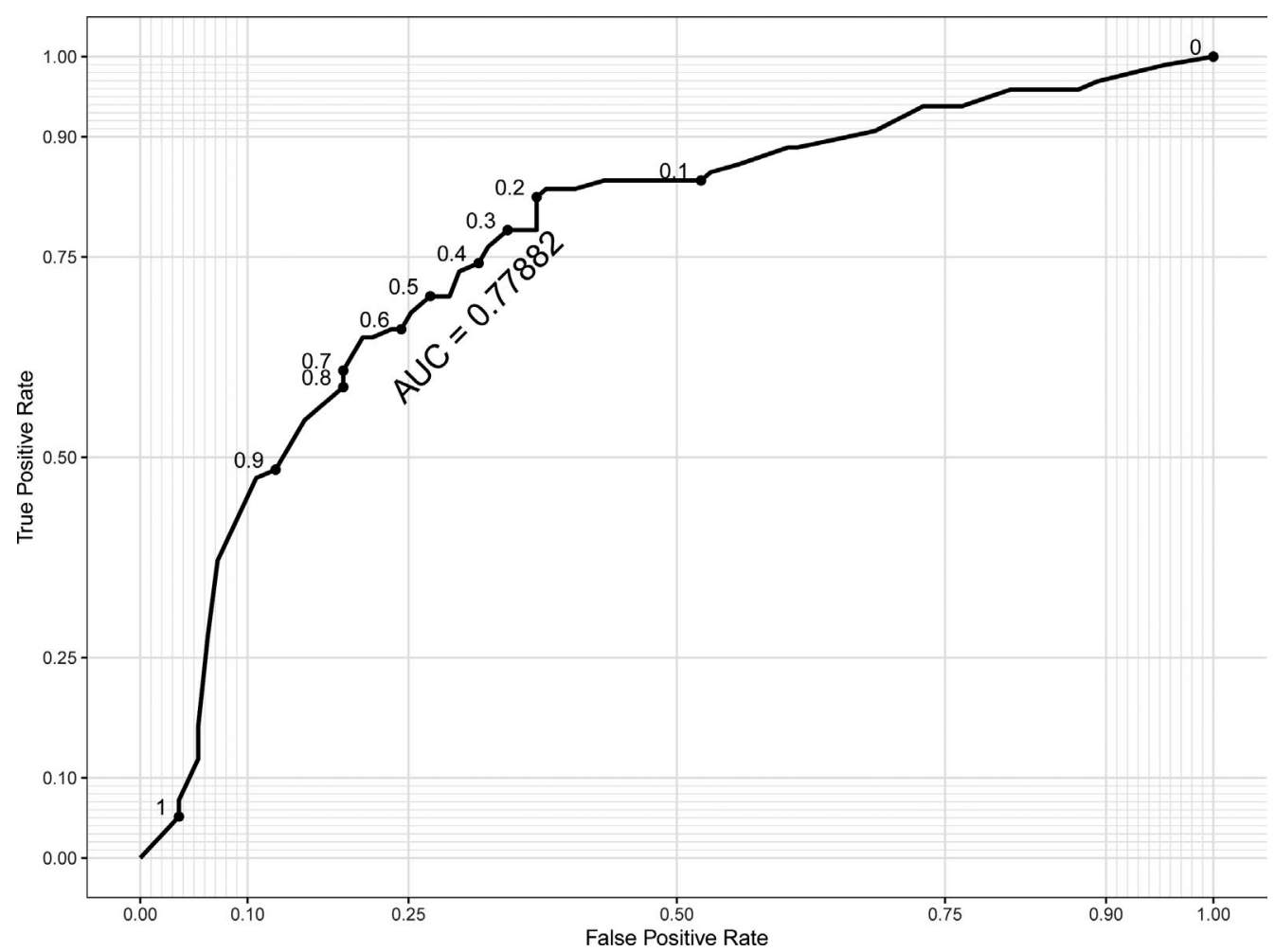

FIGURE 3 ROC curve with different thresholds

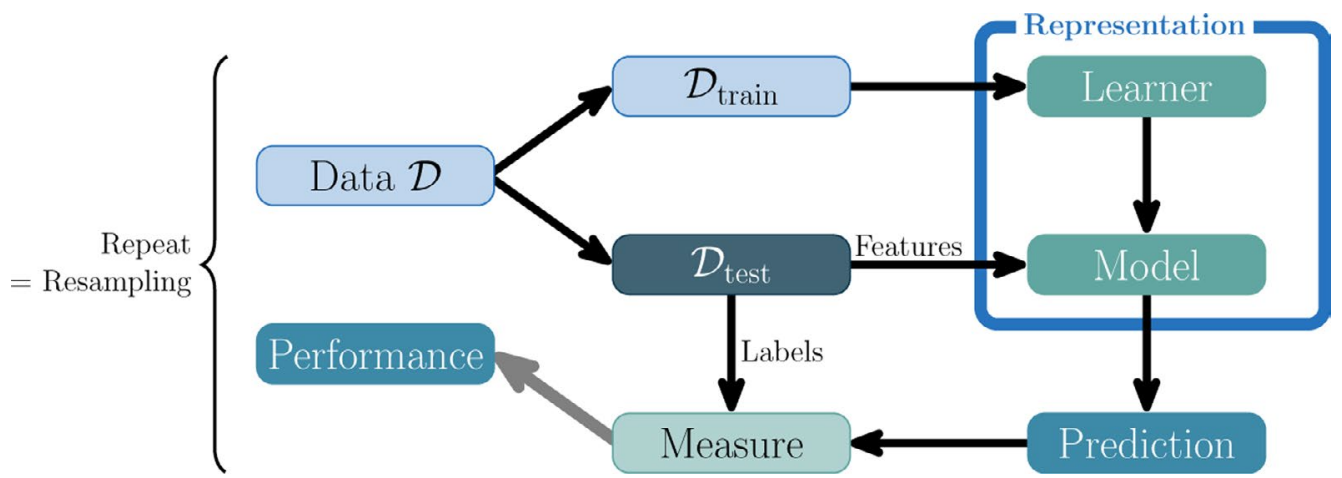

FIG URE 4 Workflow of machine learning modeling: The dataset $D$ is split into a train and test set. The learner fits a model on the training data. The learner and the resulting model are the representation of the learning process. The resulting model predicts the target variable in the test data. Its performance is then evaluated on those predictions given a performance measure. The repetitive application of this procedure is called resampling

\section{Bias and variance}

Bias and variance are concepts closely related to model complexity. The bias of a model quantifies its tendency to be 'systematically off' with respect to the true underlying functional relationship in the data. Bias is closely connected to structural assumptions in the model 


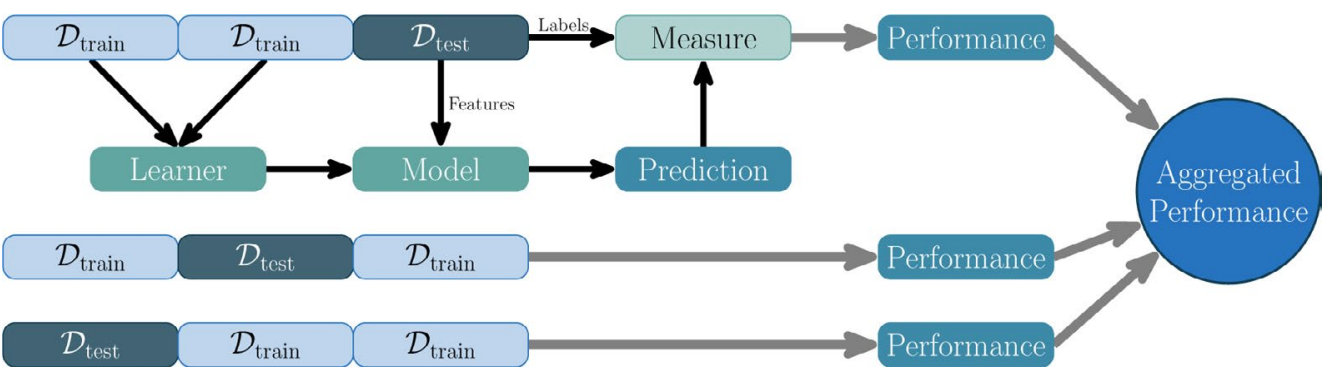

FIGURE 5 Example of a threefold cross-validation. The dataset $D$ is split into three equally sized subsets. The fitting and evaluation procedure of the model is repeated three times, with a different subset serving as training sample each time. The performances of the three test sets are aggregated to a final score. (a) Polynomial models with different degrees fitted to the training data. (b) Difference between train and test error for polynomials of different degrees
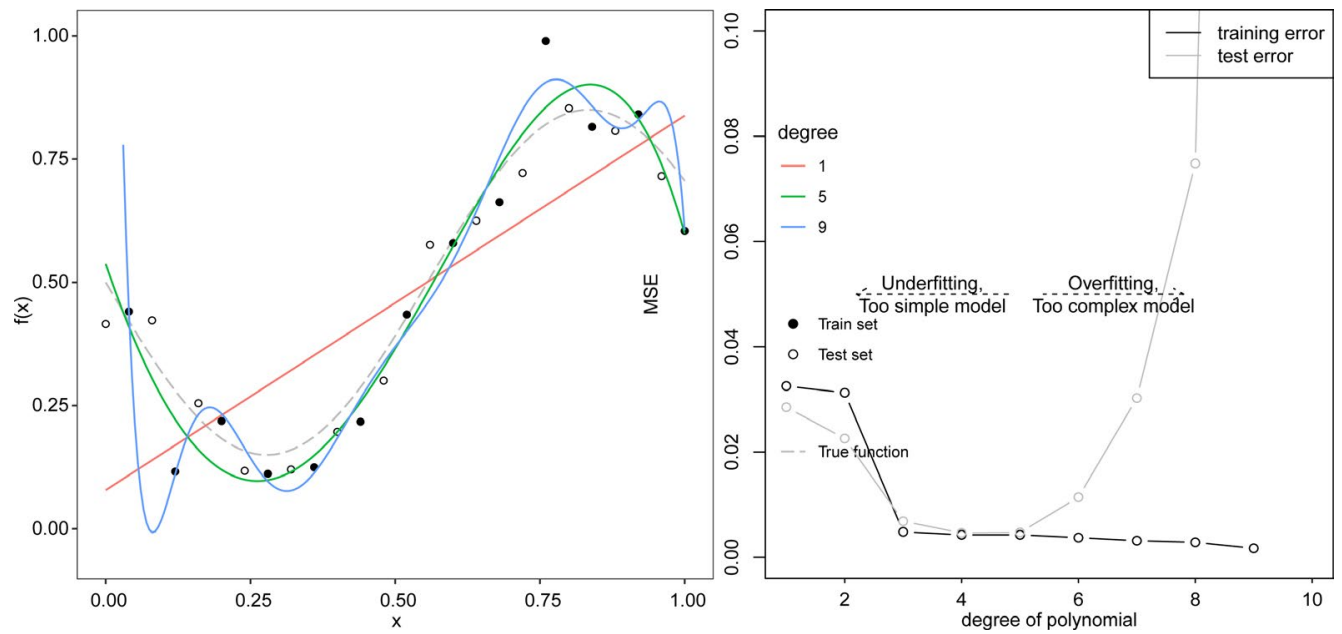

FIG URE 6 Figure (a) shows regressions of different polynomial degrees: too simple models "underfit" the data while too complex models tend to "overfit". Figure (b) shows the performance with test data, as quantified by the means squared error, for different polynomials of different degrees. After a degree of five, the performance with the test data worsens the more the polynomial degree increases. This illustrates how overfitting to the training data results in inferior predictive performance. The figure is taken from (Bischl, 2020)

class (e.g., being linear in the raw features) and therefore also to the model's tendency to underfit. The variance of a model quantifies its tendency to vary, when the same learner is fitted on similar different subsamples from the same underlying distribution. High variance implies that a model fluctuates strongly under this resampling process and is therefore unstable.

It can be shown that the generalisation error in supervised ML can be partitioned into three components: squared bias, variance and irreducible noise of the underlying distribution. Less complex models exhibit a stronger bias but less variance, whereas the opposite holds for more complex models and their hypothesis spaces. A strong bias implies that, if the bias of the model matches the properties of the underlying data distribution and true functional relationship in the data, it can be learned efficiently despite sample sizes and still generalise well. If the bias does not match the properties of the true functional relationship, the model is doomed to underfit. Again, a linear model serves as a good example: if the 
underlying relationship is truly linear, the model may learn it even when the data are sparse. This can be advantageous in studies with small samples, such as the analysis of teaching quality with questionnaires in a school. However, if the true relationship has a negligible linear component, the model learns mostly noise, even with much data, such as when analysing large MOOC datasets. More complex hypothesis spaces with larger variance but small bias carry a lower risk of underfitting, but usually need larger sample sizes to drive down the error component due to their variance (e.g., a deep neural network).

A reasonable question is how this theoretical understanding can be exploited to minimise generalisation error. As bias and variance are somewhat at odds with each other, it is necessary to find the best possible trade-off between the two. Usually, this is achieved through model comparison via cross-validation. A tendency towards simpler models in $\mathrm{ML}$, to exploit their better properties to efficiently work with smaller sample sizes, is sometimes called structural regularisation. This is also part of the reason (among several other advantages) why we recommend in the section 'Model classes' to gradually move from less complex models to more complex ones when trying out ML models on a new task.

Model parameters can be similar between subsamples (i.e., the variance is small), but the model will probably be an inadequate representation of the true underlying data structure. Because avoiding ill-fitting models was part of the reason for applying $\mathrm{ML}$ in the first place, simply accepting high bias cannot be the way to go. Yet, if the bias is reduced by applying more flexible models to the data, the variance is typically increased, because the idiosyncratic randomness of the subsamples is modelled. This leads to very different model parameters in each subsample. In consequence, the generalisation error is high and the model will be rather unsuitable for future prediction. Thus, reducing the bias generally increases the variance and vice versa. We therefore must deal with the tradeoff between bias and variance, depicted in Figure 6, and one primary goal of ML modelling is to find the optimal model complexity for accurate prediction (low bias and variance), which is usually achieved by estimating models of different complexity and estimating the generalisation error (e.g., via cross-validation).

Flexible model types can adapt to the specific characteristics of training sets and therefore show higher variance in the prediction of test data. Inflexible models, on the other hand, are confined to their restrictions (such as linearity), meaning that their predictions typically have a high bias (Figure 7).

\section{Optimisation}

The performance of many predictive algorithms depends on so-called hyperparameters. These model-internal parameters influence the model-space of the learning algorithm or its fitting process. The penalty parameter in a regularised regression model or the number of split variables in a random forest are examples of such hyperparameters. Hyperparameters are not learned by training, but must be set in advance, by the user or by (hopefully adequate) defaults in software.

Unfortunately, hyperparameter configurations often need to vary for different tasks, with generalisable and robust default heuristics being often hard to define. The process of finding the optimal hyperparameter configuration in data-centred fashion is called tuning.

Tuning is usually conducted in a black-box optimisation fashion, see Figure 9, where configurations are evaluated by their cross-validated score (which is computationally expensive) and then iteratively improved by a derivative-free method, such as grid search, random search (see Bergstra \& Bengio, 2012), or model-based approaches (e.g., Bayesian optimisation; see Bischl et al., 2017). Whereas grid search uses every possible combination of hyperparameters in a predefined search grid, random search tries out a chosen number of 
random combinations. The latter method is typically recommended (see Bergstra \& Bengio, 2012). Model-based optimisation is more appropriate for high-dimensional search spaces and iteratively improves by trying to balance exploration and exploitation in the search process through $\mathrm{ML}$ on the inside (i.e., using a ML model to optimise the settings of another model). A review of common optimisation methods is provided in Luo (2016).

Tuning requires an additional, independent resampling loop (see Figure 8), because repeatedly evaluating all hyperparameter configurations on the same cross-validation splits would add an optimistic bias to the evaluation. The additional resampling loop is used to avoid this problem by keeping the tuning process strictly separated from the general model evaluation: the outer split is created to obtain a test set, which is only used for the final model evaluation after the tuning, and an outer training set. The outer training set is again separated into an inner test set, the inner split, which is only used to evaluate the hyperparameter configurations, and an inner training set on which the models, configured with those hyperparameter configurations, are trained. So, in summary, the inner loop is used for tuning evaluation and the outer loop for final model evaluation. Often, this technique is extended to performing two cross-validations: one on the inner resampling loop, another one on the outer (see Simon, 2007). This is called nested cross-validation and visualised in Figure 8.

\section{Preprocessing}

While there are many types and sources of raw, unprocessed data, the collected data usually cannot be analysed without preparation. Just like in classical statistical analyses, the raw data needs to be cleaned during the data preprocessing step. The preparation of datasets is neither a novel process for most educational researchers nor unique to ML. For example, various data transformations to meet particular model assumptions have been developed and proposed in the fields of psychology, education and economics, such as log-transformations or the Box-Cox transformation (Osborne, 2010). (However, there are

\section{Low Variance}

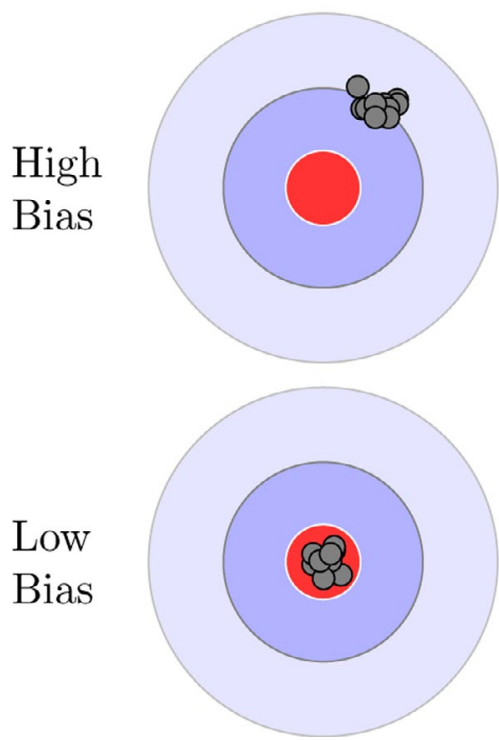

High Variance

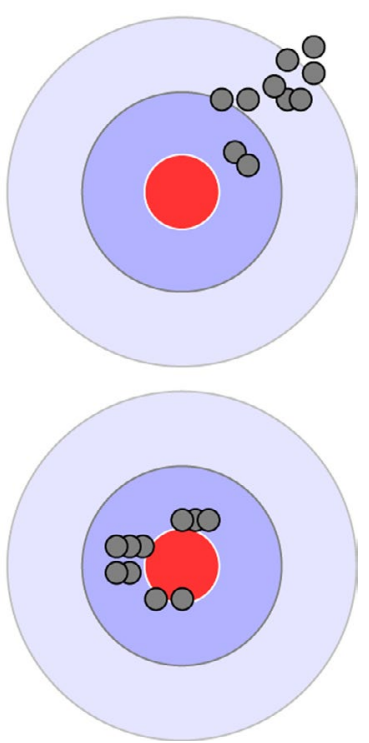


several important arguments for a thoughtful and careful use of the transformations and fitting the adequate models instead of transforming the data in order to use, for example, linear regressions; e.g., O'Hara \& Kotze, 2010; Osborne, 2002.)

The complexity of the data, as for example in sensor data or other digital footprints, makes it an integral part of ML modelling, which should be integrated in the resampling process, described in the section 'Resampling'. This is particularly relevant if data-driven decisions on the complete dataset have to be made during preprocessing (e.g., dimensionality reduction or transformations). In general, preprocessing is a complex and often cumbersome procedure. However, major parts including the handling of missings, the checking for feasible values (technically and content-wise), or the processing of different formats (e.g., time or text) are recurring problems.
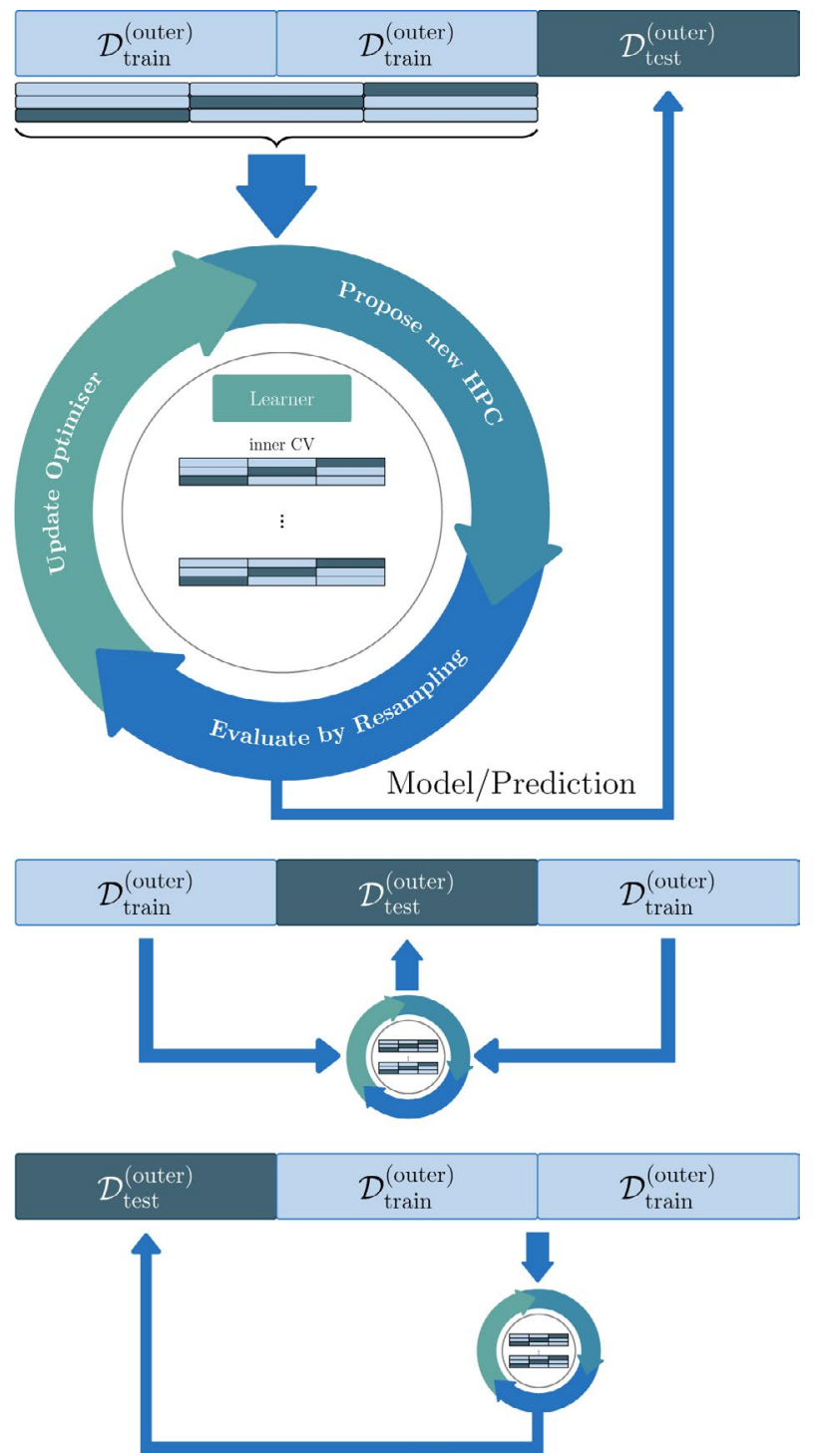

FIG URE 8 Nested cross-validation with three inner and three outer folds used for tuning: Each hyperparameter configuration (HPC) is evaluated on an inner cross-validation fold, while the resulting tuned model is evaluated on the outer loop. $\mathrm{D}$ denotes the data 


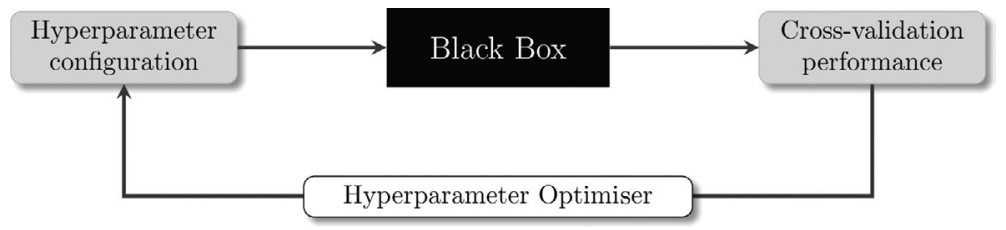

FIGURE 9 Hyperparameter tuning: A hyperparameter configuration is proposed, the enters the model, which is evaluated through resampling. The Optimizer receives the performance, adapts the hyperparameters and the next iteration starts. (a) Permutation Importance (b) Partial Dependence Plot

Regardless of the different preprocessing methods, an Exploratory Data Analysis (EDA) should be started in univariate fashion and extended to bi- and multivariate approaches. The univariate analysis should include a histogram or boxplot for numeric variables as a first visual indicator as well as summary statistics including the mean, the median, and also the minimal and maximal values. Moreover, the number of missing values might need special treatment. When looking at the created plots, one should always check whether the given values are plausible. While a student's IQ of 153 might be rare but still possible, a human age of -77 or 999 years is not. Detecting and resolving such inconsistencies within the data can be crucial for the later analysis.

As in classical statistics, typical preprocessing steps in ML are

- handling of missing values including the detection of hidden ones,

- encoding of categorical variables (factor encoding), and

- dealing with imbalances in the target variable.

How much preprocessing is needed strongly depends on the targeted ML algorithms. A tree-based method, for example, can naturally deal with missing (through transformation) or categorical values (the latter are also called factors). Hence, there is usually no need to worry about imputation (of missing values) or factor encoding. Other algorithms, like Gaussian processes or k-nearest neighbours, are based on numeric distances. As a result, character strings or categorical variables need to be transformed into numeric values. Although this can be achieved in different ways, dummy- or one-hot-encoding is probably the most common approach. Its idea is to replace the original categorical variable with individual binary-coded variables. Each of the categories is transformed into such a binary variable, except for one, which serves as the reference category. An additional aspect to consider in educational data is the granularity of the level, such as the analysis on the keystroke level or values aggregated by session, student, classroom or school (see Romero \& Ventura, 2013).

Also, especially in educational research, the 'art' of imputing missing values is still a current research topic. There are procedures of different complexity, depending not only on the learner (i.e., the untrained model) but also on the type of variable containing the missing values; that is, whether they are numerical or categorical values that must be imputed. For numeric variables, missing values may be imputed by the mean or the median value of the variable. More complex methods take the distribution into account (histogram imputation) or use an additional ML model to predict the missing values based on the other variables in the dataset. For tree-based methods, it is also common to 'separate' the missing values by imputing them with outlying values (i.e., $2 \times \max \left(x_{k}\right)$ ), to help the tree to separate these from the other data. Doing so allows the tree to easily distinguish between the missing and other values. For categorical variables, this separation is trivially possible by creating a new category for the missing values. As mentioned above, missing values can be predicted by an additional model, created exactly for this purpose. 
Typically, a whole set of the preprocessing steps is needed to solve a task. Since the steps are usually executed in serial fashion, the process is often called pipeline. In general, it is important to implement a modelling pipeline directly into the resampling procedures, described in the section 'Resampling'. This standardised procedure ensures that unbiased estimates of the model's generalisation error can be obtained. Since a wide range of preprocessing options exists to deal with certain situations, different choices lead to different pipelines. Hence, to find the optimal pipeline for the given task, tuning can be used.

\section{Prediction and explanation}

In an influential article, Breiman et al. (2001b) proposed two general philosophies of statistical modelling: a data modelling culture, assuming a prespecified (true) theoretical model for the distribution (e.g., the assumption of a linear relationship between variables $X$ and $Y$ in the population) and an algorithmic modelling culture, treating the distribution as potentially complex and unknown. Today, the distinction of these two approaches to statistical modelling has become blurred. However, intuitively, it seems clear that the first direction appears preferable from a theory-driven point of view, which is prevalent in many behavioural sciences, such as psychology (e.g., Haig, 2019), sociology (Ritzer \& Stepnisky, 2020), or the educational sciences (e.g., Kunter, Baumert, et al., 2013; Wulf, 2003). However, even in classical theory-driven sciences, the inclusion of data-driven ML methods has already led to huge advances in both understanding and predicting the phenomena of interest. In linguistics, for example, researchers have been struggling for decades to unravel how humans produce speech-a topic that is particularly relevant for education with its focus on communication. Only recently, with the release of latest ML-based language models (e.g., transformers: RoBERTa, BERT, GPT-3), computers can produce almost human-like language with enough training data (Solaiman et al., 2019). Keeping in mind that ML models are particularly popular in economic contexts, it is reasonable to assume that ML emphasises out-of-sample prediction and cross-validation so much, because it was designed to work and generate predictions with an actual (often financial) merit.

As we have discussed in the previous sections, model quality is operationalised somewhat differently in ML compared to classical statistics. In the classical statistical approach typically taken in the educational sciences, models are rarely tested for their performance on new data. The term 'prediction' is somewhat misleading in the way it is used in the context of classical in-sample modelling, because the predicted data has already been used to fit the model. Whereas the terms prediction and estimation are often used interchangeably, predictions in the true meaning of the word, are made for unknown (future) data (so, a bit provocatively, it could be argued that 'postdiction' may be a more fitting term for estimation). The same notion holds true for the evaluation of model performance. Estimating the performance of a model on the same data it was used to fit will likely lead to an overestimated predictive performance on new data (see, e.g., Stachl, Pargent, et al., 2020; Yarkoni \& Westfall, 2017). This is particularly relevant for analyses that include many predictors such as TIMSS, PISA, MT21 or TALIS, which typically use enormous assessment batteries (see the section 'Voluminous data'). Even though-due to convergence problems in classical statistical models - these variables never enter the analysis together, the large number of individual publications based on these large-scale studies draws a strongly biased picture of the actual predictive power of the derived models. This is problematic for education, because these publications often trigger changes in educational systems, for example in the teacher education or school structures, and should therefore be reliable and unbiased.

Theoretically elegant models with limited predictive capacity are prone to impede scientific progress and may lead to models that-in the worst case-ignore highly salient patterns in 
the data (Shmueli, 2010; Yarkoni \& Westfall, 2017). Importantly, emphasising the predictive validity of models does not oppose explanatory value but rather complements it and can in some cases even prevent erroneous explanations. As visible in Figure 6, simply fitting a flexible model to the data results in an overestimation of the amount of variance the model can explain. Thus, the explanatory and the predictive capacities of the model are impaired. This brings us to another important topic, strongly tied to the future of the educational sciences: replicability. Many published scientific results, especially in the field of behavioural sciences and most prominently (social) psychology, have shown to be not replicable (Collaboration et al., 2015; Klein et al., 2018), meaning that they do not actually apply in the general population and therefore are scientifically worthless.

Reasons for this lack of replicability are manifold: because positive results tend to appear more 'exciting', they are more likely to be published, so that the resulting pattern of published results is strongly biased (the so-called 'publication bias'). Since publications and impact factors of the publishing journals are major currencies in the academic system (see Brembs et al., 2013, for an interesting perspective), coincidentally positive results are not outweighed by negative results, as those stay in the file drawer (Ioannidis, 2005). Also, empirical researchers tend to apply questionable research practices, such as cherry picking of results, optional stopping during the assessment when the results just happen to support the hypothesis, selective removal of cases, deliberate inclusion and exclusion of covariates, and many others, by now widely known under the term $p$-hacking (Simmons et al., 2011). John et al. (2012) showed that most researchers in their sample reported to have applied at least one questionable research practice, oftentimes without being aware of any misconduct. This leads to many underpowered studies in the behavioural sciences and results in the observed lack of replicability (Higginson \& Munafò, 2016; Maxwell, 2004; Schoenfeld, 2018).

Relating these practices to ML, Yarkoni and Westfall (2017) described $p$-hacking as 'procedural overfitting', thereby highlighting that the process of applying questionable research practices lead to an overfit to the data and therefore to a lack of replicability. Even though a stronger focus on prediction does not rule out questionable research practices, the process of selecting the best-fitting model to predict novel data at least prevents the process of using these practices to produce an overfit to the training data. As shown in the previous sections, overfitting the model to the training data worsens the performance for the test sample: the result obtained in the training sample cannot be replicated with the test sample. Also, since there is no clear cutoff value, applying measures to just pass the threshold of statistical significance becomes obsolete. Notably, this idea has already been brought forward as 'bring your own $\alpha$ ' for classical (frequentist) analyses (Lakens et al., 2018). Thus, a predictionbased focus in modelling will not end the replication crisis and an exhaustive discussion of this topic goes far beyond the scope of this review, but it may surely help paving the way for a more reliable empirical science in the field of education.

\section{Interpretable machine learning}

Despite the prediction-centred approach of $\mathrm{ML}$, researchers have developed several approaches to better understanding the inner workings of models and to quantify the impact and interactions of features. Model explanation is particularly important in flexible black-box models (e.g., ensembles), that do not offer model coefficients (e.g., beta-coefficients in linear regression) themselves (see Biecek \& Burzykowski, 2021; Molnar, 2020, for a good overview). For the educational sciences, model interpretation or explanation is crucial, because improving education is a continuous task for societies. Hence, information on the relationships between individual variables is key for pushing the right buttons to achieve positive consequences in this context. Also, model interpretation is essential to evaluate algorithmic fairness and model 
security (e.g., adversarial attacks in essay grading or privacy protection), topics that are covered in subsequent sections. Although not always straightforward (e.g., as the estimation of standardised weight parameters in linear regression models), the importance of variables can be quantified using auxiliary techniques (e.g., Breiman, 2001a, 2001b). These include permuting the values of each feature and observing the related decrease in model performance after predicting on the permuted data, as depicted in Figure 10a. The use of visualisations can also be enlightening, especially for potential inferences about causality (see, Zhao \& Hastie, 2019). The following sections will report on some of the most common techniques of IML. However, interpretable machine learning is a relatively young and vivid field of research and novel methods are continuously developed. Moreover, if applied incorrectly, various pitfalls of ML model interpretation lurk, such as erroneous model generalisation, dependent features, feature interactions or unjustified causal interpretations. An overview for the practitioner on common IML pitfalls and solutions on how to avoid them are presented in Molnar et al. (2020).

\section{Permutation importance}

Permutation feature importance was originally introduced by Breiman (2001a, 2001b) as a built-in feature of the random forest algorithm. The idea behind it is very intuitive: if a feature is important for the prediction and we then permute the values of this feature, the prediction performance should become weaker. However, if the prediction performance remains almost unchanged, the feature is probably unimportant. The permutation feature importance is then equal to the measured loss of predictive performance between the predictions for the original data and the permuted one. An example for this can be seen in Figure 10a for predicting scores of a reading test. While permutation importance was introduced with the random forest, it is a model-agnostic method and can hence be used irrespective of the applied learning algorithm. Permutation feature importance measures are sensitive to correlations between predictors, causing highly correlated features to appear relatively disadvantaged in the feature ranking. This aspect does reflect the reality of the model (i.e., correlated predictors are only used in a subset of the trees in the forest) but can complicate interpretation if the potential unique contribution of a feature is of interest. Alternative methods (Strobl et al., 2008) have been proposed to quantify the importance of predictors regarding their correlation with other features.

\section{Partial dependence plots}

Partial dependence (PD) plots show the marginal effect of a single feature on the target variable, when integrating over all other covariates (Friedman, 2001). An example of a PD plot is depicted in Figure 10b. In other words, the plot shows the average predictions for the target variable under the assumption that the value in one or two features had a certain value, not accounting for correlations with other features and interactive effects. PD plots can also be computed for selected pairs of features and then represented as a $2 D$ heatmap or a 3D surface plot.

\section{Individual conditional expectation plots}

On the local level (i.e., for individual cases), individual conditional expectation (ICE) or ceteris paribus (CP) plots show the change in the predicted value for a single feature, when conditioned on a specific observation (e.g., for one measure obtained from a single student). Therefore, all remaining features are held constant to the observed values, and predictions 

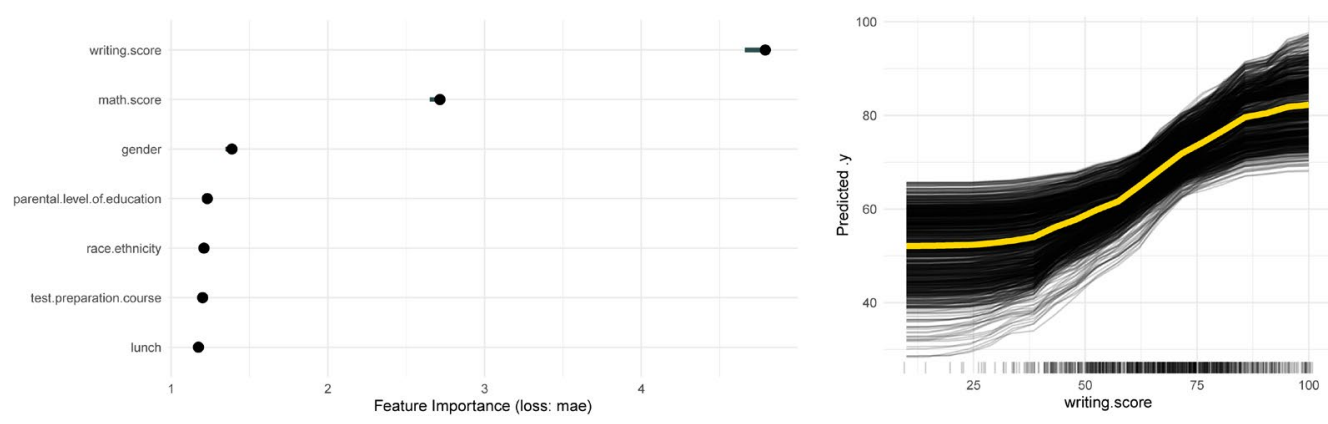

F I G URE 10 (a) Permutation Feature Importance: The score in a reading test is predicted by seven features, depicted on the $y$-axis: writing.score = the score in a writing test; math.score = the score in a mathematics test; gender = the gender of the student; parental.level.of.education = the parents' highest academic degree or non-academic degree qualification; race.ethnicity = the ethnicity of the student; test. preparation.course $=$ a dummy variable indicating the completion/ non-completion of a test preparation course; lunch $=$ the entitlement $/$ non-entitlement to a free or reduced lunch. The $x$ axis depicts the deterioration in the mean absolute error following the permutation of the values of each feature. (b) PDP of a random forest Regression model. It shows the effect of the covariate writing score (writing.score, $x$ axis) on the obtained reading score of $n=1000$ children. (b) shows the marginal effect in the foreground (yellow) as well as the ICEs as individual lines in the background (grey)

are calculated simply by varying the covariate under consideration. ICE plots for a specific feature are typically computed for all observations in a data set and plotted together. It can be shown that the average of all ICE curves coincides with the PD curve (Goldstein et al., 2015). In addition to PD plots, ICEs can be helpful to identify interactions between features in the dataset. Interactions can be indicated by diverging lines in the ICE plot. If all lines are parallel, usually a homogeneous effect of the covariate is assumed. Figure 10b shows the individual relationships between writing scores and the predicted reading scores in the PD plot in the background of the integrated PD line.

\section{Counterfactual explanations}

In contrast to previously introduced methods, so-called local interpretation methods which try to explain single predictions such as 'why did student $A$ receive this score in a writing test based on these feature values?' Wachter et al. (2017) proposed to use counterfactuals to describe single predictions. A counterfactual explanation describes a causal situation of the form 'if $X$ had not occurred, $Y$ would not have occurred'. In other words, it describes the smallest change to the feature values that changes the prediction to a predefined output. Additionally, a counterfactual explanation should meet the following criteria (Molnar, 2020): it should produce the predefined prediction as closely as possible, be as similar as possible to the instance regarding feature values, change as few features as possible and have feature values that are plausible. Whereas Wachter et al. (2017) use a weighted combination of the first two criteria as optimisation function to determine counterfactuals, Dandl et al. (2020) suggest to simultaneously optimise those four criteria by multi-objective optimisation using the Nondominated Sorting Genetic Algorithm or short NSGA-II by Deb et al. (2002).

\section{Machine learning and fairness}

Fairness is one of the central topics in educational sciences (Mashek \& Hammer, 2011). It must be considered in standardised assessment (such as standardised achievement tests) 
and when it comes to unstandardised but complex decision making (such as assignment to different school types). Unfairness may root in unfair assessment, but unequal (educational) opportunities can also occur due to real differences in achievement of, for example, different social groups (Gipps \& Stobart, 2009). Deeper social inequalities are discussed as the origin of these actual differences in performance (Mashek \& Hammer, 2011). ML can detect both kinds of inequalities. In terms of unfair, biased assessment, it offers promising tools and methods to reduce or even eliminate the influence of discriminating characteristics in assessment (e.g., Kusner \& Loftus, 2020). In terms of inequalities that root in social structures, it can reveal relevant characteristics and therefore offer valuable insights for promotion programmes and special training. This can be achieved, for example, by using online data to identify behaviour and personal characteristics that lead to course dropout (Lykourentzou et al., 2009). At the same time, the unreflected use of ML models can perpetuate and amplify biases in the training data (e.g., existing social disadvantages) that can lead to algorithmic bias and discrimination (Buolamwini, 2018, e.g., by race).

\section{Fairness in educational assessment}

Fairness has been a sensitive topic in educational assessment for a long time (e.g., OECD, 2019b). Comparisons along different demographic dimensions, like social status, gender or migration status, reveal broad and continuous discrepancies in achievement (for examples see further analyses of PISA, TIMSS or PIRLS, Entorf \& Minoiu, 2005; Schubert \& Becker, 2010) and the social sciences offer several theories on how these discrepancies emerge and how they are ignored (Feagin, 1992; Henrich et al., 2010). In standardised assessment, individual achievement is typically referenced to a norm group. Accounting for well-known discrepancies in test scores, different norm tables are available for groups, such as male and female students, sometimes for students with a different mother tongue, or for people with divergent educational backgrounds (Nitko, 1996). This practice can at least account for linear shifts in the mapping of correctly answered items and test score. However, decision making in education is typically more complex, less comprehensible, and less standardised than a performance test: everyday fairness in evaluation and grading mainly depends on individual teachers. Even though biases in unstandardised assessments are discussed in teacher education, there are hardly any structures in practice that aim at monitoring fairness in daily school life or educational decision making (Brookhart, 1993). It is therefore not surprising that empirical research persistently reports discrimination in the assessment of academic achievement and regarding access to higher education (for examples, see Freeman, 2009; Ready \& Wright, 2011). Most studies cited above used linear regression or covariance analyses to detect the dependencies of achievement and demographic characteristics. With these types of statistical analyses, only linear dependencies can be detected. Yet, discrimination is often inconspicuous and manifests itself through complex interactions of different predictors-reliable analyses must account for this complexity. We therefore plead for the use of $\mathrm{ML}$ for the analyses of inequalities in educational opportunities and the educational sciences in general. We first discuss pitfalls in the application of ML methods that can lead to or enhance unfairness. Second, we illustrate how the deliberate application of ML can be used to detect and to minimise inequalities in educational assessment and decision making.

\section{Pitfalls to fair machine learning}

In many cases, the application of $\mathrm{ML}$ algorithms has demonstrated the potential to produce and enhance existing inequalities. Hidden feedback loops, for example, are a well-known 
problem in the context of ML applications. They occur when the application of an algorithm influences the data it uses to learn and may result in plain computational bugs, such as endlessly increasing font sizes (Sculley et al., 2014), but can also deepen social inequalities when ML algorithms are applied in critical contexts (Kusner \& Loftus, 2020). This is the case, particularly, when sub-populations and underrepresented groups are discriminated by the algorithm (see Liu et al., 2018). In this sense, hidden feedback loops can undermine the potential benefits of predictive models, by perpetuating existing inequalities and biases.

In a recent example of algorithmic unfairness, predictive grading was used in colleges in the United Kingdom to substitute for cancelled exams, due to the COVID-19 pandemic (Porter, 2020). The algorithm used the ranking of students within their school and the school's historical performance as predictors for the prediction of the grades. Unfortunately, the school's historical performance (and therefore the predicted grades) was highly correlated with socioeconomic status of the students: for the same performance on an individual level, students in rich colleges received better grades compared to those in poorer ones. In another instance, the evaluation of a teacher's performance was based on their students' past performance and the degree to which their grades exceeded the expectations (Strauss, 2015). This approach, however, led to the absurd case that already very good students would cause the teacher's performance evaluation to drop (i.e., students could not exceed the very best grades they were already having). These examples highlight that algorithmic transparency is crucial and call for a rigorous science of IML. These calls have become loud and frequent (Doshi-Velez \& Kim, 2017) and it will be a constant challenge to ensure that ML models are strictly in line with ethical values (Irving \& Askell, 2019).

The topic is especially relevant in education, because multiple inequalities are already present in educational systems (Freeman, 2009; Mashek \& Hammer, 2011). If, for example, an algorithm predicts a low retention probability for a certain group of students and teachers therefore stop fostering these students, because they believe it to be pointless, the probability of retention becomes even lower for this group-the negative feedback loop is complete. This phenomenon is well-known under the term 'Pygmalion effect' (see Friedrich et al., 2015).

\section{Machine learning for fairness}

Despite the issues discussed above, ML models can also be effectively used to enhance fairness. For a first argument, we propose to use ML to detect existing inequalities in assessment tools, like standardised tests. Predicting gender or migration status from single items should not be possible-not even with complex non-linear ML algorithms. If, however, we are able to do so, we can use the techniques described in the IML section to detect the sources of inequalities and revise the items that caused the unfairness.

Second, a paradigm shift towards prediction also comes with a reconsideration of predictor variables, thus, consequences for the feature selection. Former studies regularly used demographic data to 'explain' unequal performances in different demographic groups (for example, Freeman, 2009; Reilly et al., 2019). In the context of ML, recent research has shown: algorithms mining through large amounts of data can be scarily successful at predicting highly sensitive personal attributes, such as sexual orientation, ethnicity or political as well as religious views (Kosinski et al., 2013), which can be readily used for discrimination. Kotsiantis (2012) demonstrated almost a decade ago that demographic data could also be used to predict students' performance-a possibility that is surely tempting but leads down a dangerous path, as shown above. To counteract this, sophisticated algorithms have been proposed to produce fair ML models. These fair ML models are able to include demographic information and avoid vicious feedback loops at the same time (Kilbertus et al., 
2020; Kusner et al., 2017). Still, this field of research is very young and a state-of-the-art solution is yet to be found (Corbett-Davies \& Goel, 2018). Nonetheless, we stress that for ML models for decision-making with consequences for individuals, fairness of ML models must be investigated and assured.

\section{Machine learning and privacy protection}

Finally, privacy protection is an aspect that deserves attention, as ML in the educational sciences is currently leaving the stage of single research projects behind and becoming part of public applications and shared data projects. In the past, privacy protection in educational research was mainly insured by anonymisation of the data (Aggarwal, 2005), which was securely stored and deleted after a certain period of time. Yet, in recent years, a large number of public data repositories and MOOCs storing educational data has emerged and continues to grow. One example is the Pittsburgh Science of Learning Center (PSLC) DataShop (Koedinger et al., 2008), which makes substantial quantities of data from a variety of online learning environments available to researchers worldwide. About $14 \%$ of the papers published in the conferences of educational data mining EDM2008 and EDM2009 utilised publicly available data from the PSLC DataShop (Baker \& Yacef, 2009). Although open data initiatives are commendable, this development also highlights that different kinds of privacy threats have become highly relevant.

Today, the most relevant threats to privacy in the field of ML in the educational sciences seem to be membership inferences (identifying if data of individual persons are part of the datasets, which is particularly problematic for school data) and model memorisation attacks (aiming at reproducing properties/features of the original dataset from a model; Rigaki \& Garcia, 2020).

One way to avoid these problems is to have models trained in distributed fashion. Instead of merging data on a centralised server, it can be partly analysed on the users' own devices. This can ensure that private information, such as GPS data, is not stored anywhere but on the users' own computers (or phone, tablet, etc.). Another way is to aggregate data on the users' devices and only send aggregate data to the server for analysis. This technique, of course, must correspond with the granularity of the analysed data (see the section 'Preprocessing').

A measure to prevent membership inferences is the application of differential privacy measures. Differential privacy usually means intentionally inflicting noise in part of the $\mathrm{ML}$ process. The noise can be added to the raw data, the loss function or, even in the outcome model parameters (Liu et al., 2021). As measures of differential privacy sometimes reduce model accuracy in a non-acceptable way, encryption of the data can be an alternative. However, encryption of the data makes it harder to compute, tune and interpret models (Tanuwidjaja et al., 2020).

\section{Construct validation}

Latent constructs play a central role in the educational sciences. Large-scale studies have typically resulted in various models based on latent variables, such as the COACTIV (Cognitive Activation in the Classroom) study, which has been conducted to investigate teachers' competencies and different domains of professional knowledge with regards to their effects on teaching quality and on students' performance (Kunter, Baumert, et al., 2013; Kunter, Klusmann, et al., 2013). The application of questionnaires makes the modelling of latent constructs inevitable in many research settings. Latent variables can be useful, 
because the overreliance on a single parameter in item selection typically leads to a narrow focus and undesirable psychometric properties. However, these latent constructs are inherently theoretical and must be rigorously validated. Even though $\mathrm{ML}$ is mostly known for identifying strictly empirical associations and its often black box-like nature (Zhao \& Hastie, 2019), it can provide important contributions to the field of construct validation and thereby be of great help for the educational sciences.

The analysis of digital footprints or smartphone usage have been extensively shown to be related to latent psychological constructs, such as the Big Five personality traits (see Harari et al., 2019; Stachl, Au, et al., 2020; Stachl et al., 2017). While this field of research used to be exploratory in nature, it has become clear that these enormous exhibits of human behaviour can be far more than just interesting correlates of established questionnaires. They can serve as an important source for the validation of latent constructs (Bleidorn \& Hopwood, 2019). Youyou et al. (2015), for example, showed that ML algorithms can use digital footprints in social media data to predict self-reported personality more accurately compared to predictions based on peer-ratings, if enough data is available. Moreover, a study by Park et al. (2015) showed that the assessment of social media language added incremental validity over classical informant reports and discriminated reliably between personality traits. Finally, researchers are becoming more comfortable with the idea that these rich representations of behaviour could not only be considered as manifestations of presumably underlying latent traits but could be used to represent individual differences themselves (Boyd et al., 2020).

As described in the section 'Educational (Big) Data', the available digital footprints for construct validation in the educational sciences are manifold. The possibilities to extract records of behaviours on important variables, such as the drivers of students' performance, can be valuable to improve learning quality (Ciolacu et al., 2017). Also, motivational constructs both on the students' and on the teachers' sides are crucial in this context (Tohidi \& Jabbari, 2012) and therefore extremely relevant for the evaluation of the learning situation (Turabik \& Baskan, 2015). These constructs are prone to be addressed and validated on the various levels of digital records that carry information about students' behaviour (Ciolacu et al., 2018). Also, the assessment of social media language, as in Park et al. (2015) for the case of personality, could be a far more valid and less biased measure for students' reading and writing skills compared to a single grade. It has already been shown that compound test scores, such as the SAT score, provide incremental validity over single high school grades, when predicting future performance (Camara \& Echternacht, 2000). This only underlines how promising the analysis of broad digital records can be for the educational sciences.

Another problem that can be tackled by $\mathrm{ML}$ is the change in the validity of constructs. This phenomenon, known as concept drift in ML or as measurement invariance in psychometrics, can occur suddenly, gradually or in recurrent fashion (Lu et al., 2018). This is particularly relevant in the educational sciences, because model updating is an important factor in the standardisation, scaling and content analysis of educational tests, such as the SAT. If, for example, specific items in the SAT were used to predict academic performance in 1990, this is not necessarily the case in 2021 . Also, latent variables as predictors of classroom performance, as in the COACTIV study or studies surrounding the MTLT (Mathematics Teaching and Learning to Teach project; e.g., Hill et al., 2005; Kelcey et al., 2019; Kersting et al., 2012), may not be as valid anymore as they used to be in the 2000s, when the studies were conducted. This is because technology as well as culture are constantly evolving, and several structural changes have occurred in most educational systems during the last decade. Taken together: for digital records, a constant re-evaluation of the data streams can be a proper way to detect a drift and in the absence of constantly updated data, a broader categorisation of variables can at least establish some comparability between older and newer studies (Stachl, Pargent, et al., 2020). 
Two aspects to consider when it comes to the establishment of construct validity with ML are the more data-driven modelling approach and the addition of non-linearity that bring along important changes to scale construction. Some might argue that, similar to traditional scale construction procedures, digital indicators of a latent construct should only be included if this is clearly suggested by theory (Bleidorn \& Hopwood, 2019). Although theoretical input for scale construction is very important, an exclusive focus on theoretically derived indicators should not be a necessity for ML-based scales and could hinder the new opportunities that these methods offer (see Stachl, Pargent, et al., 2020). One of the strengths of MLbased assessments lies in the ability to model more complex relationships and interactions in the data whereas traditional scale construction efforts are usually guided by confirmatory, linear structural equation modelling procedures. ML-based methods can therefore be of help to ensure that non-linear relationships with constructs are also accounted for.

Taken together, the opportunities of ML-based approaches for construct validation are manifold, due to their focus on robust prediction (Yarkoni \& Westfall, 2017) and especially the data they can handle (Markowetz et al., 2014). The theory- and typically questionnairebased assessment of latent constructs can therefore greatly profit from these possibilities by adding incremental validity to self- and peer-ratings (Youyou et al., 2015), provide more behaviour-based measures of the constructs (Stachl et al., 2017), or even existing theories through consistent exploratory findings (Waljee et al., 2014). Negative external validity can be particularly helpful for the detection of problems with theoretical constructs and the way they were operationalised (see Bleidorn \& Hopwood, 2019). Lastly, several ML-based solutions for the decreasing validity of models have already been proposed and are prone to become more important with increasing use of digital records for educational research (Lu et al., 2018).

\section{CONCLUSION AND OUTLOOK}

The technological evolution during the last 20 years, particularly in the field of information technology, has impacted science on almost every level and resulted in a need for new data analysis techniques on the way. The widespread availability of massive amounts of data has triggered a methodological shift in behavioural research. However, the potential for the educational sciences has barely started to unfold. ML approaches are able to deal with large amounts of variables without encountering convergence problems and to learn highdimensional complex, non-linear relationships - this holds for digital Big Data and mobile sensor information from devices, such as smartphones, as well as large-scale studies.

ML can provide new perspectives on data analysis and provide a new standard for statistical models that also applies to classical research settings with smaller data samples. The prediction-based approach of $\mathrm{ML}$ in combination with resampling methods, such as $\mathrm{CV}$, may help preventing the educational sciences from stumbling into a replication crisis, as we have seen in psychological research (and many other disciplines). In addition to the ability to handle many variables and to model complex relationships, the application of ML techniques in the educational sciences can help to improve the science as a whole. As described by Yarkoni and Westfall (2017), a stronger focus on prediction neither impedes theory-driven research nor does it render all models strictly exploratory black boxes. The fields of IML and fair $\mathrm{ML}$ are concerned with cracking open the black box that $\mathrm{ML}$ is often considered to be (Ratner, 2017).

Most importantly, the rise of big, behavioural data and ML is far from over. Best highlighted by the staggering educational catastrophe, induced by the ongoing COVID-19 pandemic (Gromada et al., 2020), educational trends such as home and remote schooling, virtual and mobile assessments, and behavioural and social tracking for personalised support and interventions are here to stay and will only become more relevant in the future. In the years to come, people will need more and better access to high quality educational and learning 
resources, independent of time and location (e.g., Bakhmat et al., 2019). This goal can only be achieved by the creation of more automated, digital learning environments that take the specific needs of students into account and at the same time reduce the burden on already challenged teachers and educators (Ciolacu et al., 2017).

ML-based learning, assessment and support systems will play a central role in this development. Moreover, future education will need to diverge from a one-size-fits-all approach to learning by considering the specific needs of individuals. Mobile, sensor-based data collections and ML prediction models will finally enable the widespread implementation of personalised learning experiences and support mechanisms in education. The skilled use of ML techniques by researchers in the educational sciences will be paramount in this development. As part of this process, we expect stronger interconnections with other evolving fields that draw from the same sources of data, such as psycho-informatics with its focus on the use of smartphones and tracking data (Markowetz et al., 2014) and information science in general (Teasley, 2019).

Obviously, data mining will become even more relevant along with the ever-growing amount of educational data (see Romero \& Ventura, 2020) and result in a stronger need for IML and a general understanding of related algorithms by researchers to advance the theoretical knowledge to the same degree. Finally, data literacy is going to become crucial for teachers and scholars in general (see Gould, 2017), especially with the perspective of having to adapt to the necessities of your students without actually seeing them perform in the classroom, as we have witnessed in the current COVID-19 epidemic. In addition to an understanding of the algorithms, not only researchers but also teachers will have to be familiar with the results ML models provide for them. In short, as the digitisation and the use of $\mathrm{ML}$ become more and more prevalent in every aspect of our lives, their understanding in form and content as well as its presentation and teaching must enter the curricula as well. As this overview has shown, the growing size and complexity of datasets and the requirements for statistical models, such as unambiguousness and replicability, urge researchers in the educational sciences to become familiar with $M L$, including all its possibilities and caveats. We believe that a thorough knowledge of IML will become an integral part of the statistical education of future researchers in the field of education and urge scholars to incorporate $\mathrm{ML}$ in their teachings. For researchers, we recommend incorporating the focus on generalisability of the models. In the sense of Ockham's Razor, a simple linear association has its merits in many contexts (especially when little data is available) but should hold under cross-validation and be compared to more complex models. In this sense, the focus on replicability should be extended to a culture of open science, shared data and available analysis codes, as has been proposed in the light of the replication crisis almost a decade ago (Collaboration, 2012).

\section{CONFLICT OF INTEREST}

None of the authors has any conflict of interest.

\section{ETHICAL APPROVAL}

In our research, we have met the APA ethical standards, no ethics approval was required for this review.

\section{DATA AVAILABILITY STATEMENT}

All data and analysis codes are availlable at https://osf.io/ntre9/.

ORCID

Sven Hilbert (D) https://orcid.org/0000-0001-5808-8357 


\section{REFERENCES}

Aggarwal, C. C. (2005). On k-anonymity and the curse of dimensionality. In VLDB, 5, 901-909.

Aggarwal, C. C. (2018). Neural networks and deep learning. Springer.

Ali, M. M. (2013). Role of data mining in education sector. International Journal of Computer Science and Mobile Computing, 2(4), 374-383.

Aulck, L., Velagapudi, N., Blumenstock, J., \& West, J. (2016). Predicting student dropout in higher education. arXiv preprint arXiv:1606.06364.

Baker, R. S., \& Yacef, K. (2009). The state of educational data mining in 2009: A review and future visions. Journal of Educational Data Mining, 1(1), 3-17.

Bakhmat, N., Maksymchuk, B., Voloshyna, O., Kuzmenko, V., Matviichuuk, T., Kovalchuk, A., Martynets, L., Uchytel, I., Solovyov, V., \& Manzhos, E. (2019). Designing cloud-oriented university environment in teacher training of future physical education teachers.

Barkley, J. E., \& Lepp, A. (2016). Mobile phone use among college students is a sedentary leisure behavior which may interfere with exercise. Computers in Human Behavior, 56, 29-33. https://doi.org/10.1016/j. chb.2015.11.001

Baro, E., Degoul, S., Beuscart, R., \& Chazard, E. (2015). Toward a literature-driven definition of big data in healthcare. BioMed Research International, 639021, 1-9. https://doi.org/10.1155/2015/639021

Beach, P., \& McConnel, J. (2019). Eye tracking methodology for studying teacher learning: A review of the research. International Journal of Research \& Method in Education, 42(5), 485-501. https://doi.org/10.1080/17437 $27 X .2018 .1496415$

Bergstra, J., \& Bengio, Y. (2012). Random search for hyper-parameter optimization. The Journal of Machine Learning Research, 13(1), 281-305.

Biecek, P., \& Burzykowski, T. (2021). Explanatory model analysis. Chapman Hall/CRC.

Bischl, B. (2020). Introduction to machine learning.

Bischl, B., Mersmann, O., Trautmann, H., \& Weihs, C. (2012). Resampling methods for meta-model validation with recommendations for evolutionary computation. Evolutionary Computation, 20(2), 249-275. https://doi. org/10.1162/EVCO_a_00069

Bischl, B., Richter, J., Bossek, J., Horn, D., Thomas, J., \& Lang, M. (2017). mlrMBO: A modular framework for model-based optimization of expensive black-box functions. arxiv 1703.03373.

Bleidorn, W., \& Hopwood, C. J. (2019). Using machine learning to advance personality assessment and theory. Personality and Social Psychology Review, 23(2), 190-203. https://doi.org/10.1177/1088868318772990

Blömeke, S., Gustafsson, J., \& Shavelson, R. (2015). Beyond dichotomies competence viewed as a continuum. Zeitschrift Für Psychologie, 223(1), 3-13. https://doi.org/10.1027/2151-2604/a000194

Bowers, A. J., \& Zhou, X. (2019). Receiver operating characteristic (ROC) area under the curve (AUC): A diagnostic measure for evaluating the accuracy of predictors of education outcomes. Journal of Education for Students Placed at Risk (JESPAR), 24(1), 20-46. https://doi.org/10.1080/10824669.2018.1523734

Boyd, R. L., Pasca, P., \& Lanning, K. (2020). The personality panorama: Conceptualizing personality through big behavioural data. European Journal of Personality, 34(5), 599-612. https://doi.org/10.1002/per.2254

Breiman, L. (1996). Bagging predictors. Machine Learning, 24(2), 123-140. https://doi.org/10.1007/BF00058655

Breiman, L. (2001a). Random forests. Machine Learning, 45(1), 5-32.

Breiman, L. (2001b). Statistical modeling: The two cultures (with comments and a rejoinder by the author). Statistical Science, 16(3), 199-231. https://doi.org/10.1214/ss/1009213726

Breiman, L., Friedman, J., Olshen, R., \& Stone, C. (2017). Classification and regression trees.

Brembs, B., Button, K., \& Munafò, M. (2013). Deep impact: Unintended consequences of journal rank. Frontiers in Human Neuroscience, 7, 291. https://doi.org/10.3389/fnhum.2013.00291

Brookhart, S. M. (1993). Teachers' grading practices: Meaning and values. Journal of Educational Measurement, 30(2), 123-142. https://doi.org/10.1111/j.1745-3984.1993.tb01070.x

Bruckmaier, G., Krauss, S., Blum, W., \& Leiss, D. (2016). Measuring mathematics teachers' professional competence by using video clips (COACTIV video). ZDM, 48(1-2), 111-124. https://doi.org/10.1007/s1185 8-016-0772-1

Budimir, S., Beierle, F., Zimmermann, J., Allemand, M., Neff, P., Pryss, R., Stieger, S., Probst, T., \& Schlee, W. (2020). Frequency and duration of daily smartphone usage in relation to personality traits. Digital Psychology, 1(1), 20-28. https://doi.org/10.24989/dp.v111.1821

Buolamwini, J. (2018). Gender shades: Intersectional accuracy disparities in commercial gender classification (tech. rep.). PMLR. https://proceedings.mlr.press/v81/buolamwini18a.html

Burman, I., \& Som, S. (2019, February). Predicting students' academic performance using support vector machine. In 2019 Amity international conference on artificial intelligence (AICAI) (pp. 756-759).

Camara, W. J., \& Echternacht, G. (2000). The sat [r] $i$ and high school grades: Utility in predicting success in college research notes. 
Campbell, P. F., Nishio, M., Smith, T. M., Clark, L. M., Conant, D. L., Rust, A. H., DePiper, J. N., Frank, T. J., Griffin, M. J., \& Choi, Y. (2014). The relationship between teachers' mathematical content and pedagogical knowledge, teachers' perceptions, and student achievement. Journal for Research in Mathematics Education, 45(4), 419-459. https://doi.org/10.5951/jresematheduc.45.4.0419

Campo, M., Shiyko, M. P., \& Lichtman, S. W. (2010). Sensitivity and specificity: A review of related statistics and controversies in the context of physical therapist education. Journal of Physical Therapy Education, 24(3), 69-78. https://doi.org/10.1097/00001416-201007000-00009

Chouldechova, A., \& Roth, A. (2018). The frontiers of fairness in machine learning. arXiv preprint arXiv:1810.08810.

Ciolacu, M., Tehrani, A. F., Beer, R., \& Popp, H. (2017). Education 4.0 - Fostering student's performance with machine learning methods. In 2017 IEEE 23rd international symposium for design and technology in electronic packaging (SIITME). IEEE.

Ciolacu, M., Tehrani, A. F., Binder, L., \& Svasta, P. M. (2018). Education 4.0 - Artificial intelligence assisted higher education: Early recognition system with machine learning to support students' success. In 2018 IEEE 24 th international symposium for design and technology in electronic packaging (SIITME). IEEE.

Collaboration, O. S. (2012). An open, large-scale, collaborative effort to estimate the reproducibility of psychological science. Perspectives on Psychological Science, 7(6), 657-660.

Collaboration, O. S. (2015). Estimating the reproducibility of psychological science. Science, 349(6251).

Conati, C., Porayska-Pomsta, K., \& Mavrikis, M. (2018). Ai in education needs interpretable machine learning: Lessons from open learner modelling. arXiv preprint arXiv:1807.00154.

Corbett-Davies, S., \& Goel, S. (2018). The measure and mismeasure of fairness: A critical review of fair machine learning. arXiv preprint arXiv:1808.00023.

Cornet, V. P., \& Holden, R. J. (2018). Systematic review of smartphone-based passive sensing for health and wellbeing. Journal of Biomedical Informatics, 77, 120-132. https://doi.org/10.1016/j.jbi.2017.12.008

Cortes, C., \& Vapnik, V. (1995). Support-vector networks. Machine Learning, 20(3), 273-297. https://doi. org/10.1007/BF00994018

Đambić, G., Krajcar, M., \& Bele, D. (2016). Machine learning model for early detection of higher education students that need additional attention in introductory programming courses. International Journal of Digital Technology \& Economy, 1(1), 1-11.

Dandl, S., Molnar, C., Binder, M., \& Bischl, B. (2020). Multi-objective counterfactual explanations. In: T. Bäck (Ed.), Parallel Problem Solving from Nature - PPSN XVI (pp.448-469). Springer International Publishing, Cham. https://doi.org/10.1007/978-3-030-58112-1_31

De Mauro, A., Greco, M., \& Grimaldi, M. (2016). A formal definition of Big Data based on its essential features. Library Review, 65(3), 122-135. https://doi.org/10.1108/LR-06-2015-0061

Deb, K., Pratap, A., Agarwal, S., \& Meyarivan, T. (2002). A fast and elitist multiobjective genetic algorithm: Nsga-ii. Evolutionary Computation, IEEE Transactions on, 6, 182-197. https://doi.org/10.1109/4235.996017

Depren, S. K., Aşkın, Ö. E., \& Öz, E. (2017). Identifying the classification performances of educational data mining methods: A case study for timss. Educational Sciences: Theory \& Practice, 17(5), 1605-1623.

Domingos, P. (2012). A few useful things to know about machine learning. Communications of the ACM, 55(10), 78-87. https://doi.org/10.1145/2347736.2347755

Doshi-Velez, F., \& Kim, B. (2017). Towards a rigorous science of interpretable machine learning. arXiv preprint arXiv:1702.08608.

Drucker, H., Burges, C. J., Kaufman, L., Smola, A. J., \& Vapnik, V. (1997). Support vector regression machines. In Advances in neural information processing systems, 9, (pp. 155-161).

Eagle, N., \& Pentland, A. (2006). Reality mining: Sensing complex social systems. Personal and Ubiquitous Computing, 10(4), 255-268. https://doi.org/10.1007/s00779-005-0046-3

Entorf, H., \& Minoiu, N. (2005). What a difference immigration policy makes: A comparison of PISA scores in Europe and traditional countries of immigration. German Economic Review, 6(3), 355-376. https://doi. org/10.1111/j.1468-0475.2005.00137.x

Fauth, B., Göllner, R., Lenske, G., Praetorius, A.-K., \& Wagner, W. (2020). Who sees what? Conceptual considerations on the measurement of teaching quality from different perspectives. Zeitschrift Für Pädagogik, 66, Beiheft, 138-155.

Favaretto, M., De Clercq, E., Schneble, C. O., \& Elger, B. S. (2020). What is your definition of Big Data? Researchers' understanding of the phenomenon of the decade. PLoS One, 15(2), e0228987. https://doi. org/10.1371/journal.pone.0228987

Fawcett, T. (2006). An introduction to roc analysis. Pattern Recognition Letters, 27(8), 861-874. https://doi. org/10.1016/j.patrec.2005.10.010

Feagin, J. R. (1992). The continuing significance of racism: Discrimination against black students in white colleges. Journal of Black Studies, 22(4), 546-578. https://doi.org/10.1177/002193479202200407

Fischer, H. E., Labudde, P., Neumann, K., \& Viiri, J. (2014). Quality of instruction in physics: Comparing Finland, Switzerland and Germany. Waxmann Verlag.

Freeman, K. (2009). Race, ethnicity and gender in education. Cross-cultural understandings. Springer. 
Friedman, J. H. (2001). Greedy function approximation: A gradient boosting machine. Annals of Statistics, 29(5), 1189-1232.

Friedman, J., Hastie, T., \& Tibshirani, R. (2001). The elements of statistical learning (Vol. 1). Springer Series in Statistics New York.

Friedrich, A., Flunger, B., Nagengast, B., Jonkmann, K., \& Trautwein, U. (2015). Pygmalion effects in the classroom: Teacher expectancy effects on students' math achievement. Contemporary Educational Psychology, 41, 1-12. https://doi.org/10.1016/j.cedpsych.2014.10.006

Gipps, C., \& Stobart, G. (2009). Fairness in assessment. In J. J. Wyatt-Smith\&C.M. Cumming (Eds.), Educational assessment in the 21st century. Springer.

Goldstein, A., Kapelner, A., Bleich, J., \& Pitkin, E. (2015). Peeking inside the black box: Visualizing statistical learning with plots of individual conditional expectation. Journal of Computational and Graphical Statistics, 24(1), 44-65. https://doi.org/10.1080/10618600.2014.907095

Golino, H. F., \& Gomes, C. M. (2016). Random forest as an imputation method for education and psychology research: Its impact on item fit and difficulty of the Rasch model. International Journal of Research \& Method in Education, 39(4), 401-421. https://doi.org/10.1080/1743727X.2016.1168798

Gould, R. (2017). Data literacy is statistical literacy. Statistics Education Research Journal, 16(1), 22-25. https:// doi.org/10.52041/serj.v16i1.209

Gromada, A., Richardson, D., \& Rees, G. (2020). Childcare in a global crisis: The impact of COVID-19 on work and family life (tech. rep.). UNICEF Office of Research - Innocenti. Florence. https://www.unicef-irc.org/publi cations/1109-childcare-in-a-global-crisis-the-impact-of-covid-19-on-work-and-family-life.html

Guleria, P., \& Sood, M. (2014). Data mining in education: A review on the knowledge discovery perspective. International Journal of Data Mining \& Knowledge Management Process, 4(5), 47. https://doi.org/10.5121/ ijdkp.2014.4504

Hadioui, A., El Faddouli, N.-E., Benjelloun Touimi, Y., \& Bennani, S. (2017). Machine learning based on big data extraction of massive educational knowledge. International Journal of Emerging Technologies in Learning (iJET), 12(11), 151-167. https://doi.org/10.3991/ijet.v12i11.7460

Haig, B. D. (2019). The importance of scientific method for psychological science. Psychology, Crime \& Law, 25(6), 527-541. https://doi.org/10.1080/1068316X.2018.1557181

Hand, D., \& Till, R. (2001). A simple generalisation of the area under the roc curve for multiple class classification problems. Hand, The, 45, 171-186.

Hanley, J. A., \& McNeil, B. J. (1982). The meaning and use of the area under a receiver operating characteristic (roc) curve. Radiology, 143(1), 29-36. https://doi.org/10.1148/radiology.143.1.7063747

Hanushek, E. A., \& Woessmann, L. (2010). Education and economic growth. In P. Peterson, E. Baker, \& B. McGaw (Eds.), Economics of Education (pp.60-67). Elsevier.

Hanushek, E. A., \& Woessmann, L. (2012). Do better schools lead to more growth? Cognitive skills, economic outcomes, and causation. Journal of Economic Growth, 17(4), 267-321. https://doi.org/10.1007/s1088 7-012-9081-x

Harari, G. M., Gosling, S. D., Wang, R., Chen, F., Chen, Z., \& Campbell, A. T. (2017). Patterns of behavior change in students over an academic term: A preliminary study of activity and sociability behaviors using smartphone sensing methods. Computers in Human Behavior, 67, 129-138. https://doi.org/10.1016/j.chb.2016.10.027

Harari, G. M., Müller, S. R., Aung, M. S., \& Rentfrow, P. J. (2017). Smartphone sensing methods for studying behavior in everyday life. Current Opinion in Behavioral Sciences, 18, 83-90. https://doi.org/10.1016/j. cobeha.2017.07.018

Harari, G. M., Müller, S. R., \& Gosling, S. D. (2018). Naturalistic assessment of situations using mobile sensing methods. In J. F. Rauthmann, R. A. Sherman, \& D. C. Funder (Eds.), The Oxford handbook of psychological situations (pp. 1-28). Oxford: Oxford University Press. https://doi.org/10.1093/oxfordhb/9780190263 348.013.14

Harari, G. M., Müller, S. R., Stachl, C., Wang, R., Wang, W., Bühner, M., Rentfrow, P. J., Campbell, A. T., \& Gosling, S. D. (2019). Sensing sociability: Individual differences in young adults' conversation, calling, texting, and app use behaviors in daily life. Journal of Personality and Social Psychology, 119(1), $204-228$. https://doi.org/10.1037/pspp0000245

Hattie, J. (2009). Visible learning. A synthesis of over 800 meta-analyses relating to achievement. Routledge.

Heine, J., \& Reiss, K. (2019). PISA 2018 - Die methodologie. In K. Reiss, M. Weis, E. Klieme, \& O. Köller (Eds.), PISA 2018: Grundbildung im internationalen Vergleich (pp. 241-258). Waxmann Verlag.

Hellas, A., Ihantola, P., Petersen, A., Ajanovski, V. V., Gutica, M., Hynninen, T., \& Liao, S. N. (2018). Predicting academic performance: A systematic literature review. In Proceedings companion of the 23rd annual ACM conference on innovation and technology in computer science education (pp. 175-199).

Henrich, J., Heine, S. J., \& Norenzayan, A. (2010). The weirdest people in the world? Behavioral and Brain Sciences, 33(2-3), 61-83. https://doi.org/10.1017/S0140525X0999152X

Higginson, A. D., \& Munafò, M. R. (2016). Current incentives for scientists lead to underpowered studies with erroneous conclusions. PLoS Biology, 14(11), e2000995. https://doi.org/10.1371/journal.pbio.2000995 
Hill, H. C., Rowan, B., \& Ball, D. L. (2005). Effects of teachers' mathematical knowledge for teaching on student achievement. American Educational Research Journal, 42(2), 371-406. https://doi.org/10.3102/0002831204 2002371

Hoerl, A. E., \& Kennard, R. W. (1970). Ridge regression: Biased estimation for nonorthogonal problems. Technometrics, 12(1), 55-67. https://doi.org/10.1080/00401706.1970.10488634

Ingvarson, L., Schwille, J., Tatto, M. T., Rowley, G., Peck, R., \& Senk, S. L. (2013). An analysis of teacher education context, structure, and quality-assurance arrangements in teds-m countries: Findings from the IEA teacher education and development study in mathematics (teds-m). ERIC.

loannidis, J. P. (2005). Why most published research findings are false. PLoS Med, 2(8), e124. https://doi. org/10.1371/journal.pmed.0020124

Irving, G., \& Askell, A. (2019). Ai safety needs social scientists. Distill, 4(2), e14. https://doi.org/10.23915/disti II.00014

Janocha, K., \& Czarnecki, W. M. (2017). On loss functions for deep neural networks in classification. arXiv preprint arXiv:1702.05659.

Jarke, J., \& Breiter, A. (2019). The datafication of education. Learning, Media and Technology, 44(1), 1-6.

Ji, Z., Lipton, Z. C., \& Elkan, C. (2014). Differential privacy and machine learning: A survey and review. arXiv preprint arXiv:1412.7584.

John, L. K., Loewenstein, G., \& Prelec, D. (2012). Measuring the prevalence of questionable research practices with incentives for truth telling. Psychological Science, 23(5), 524-532. https://doi.org/10.1177/0956797611 430953

Juhaňák, L., Zounek, J., \& Rohlíková, L. (2019). Using process mining to analyze students' quiz-taking behavior patterns in a learning management system. Computers in Human Behavior, 92, 496-506. https://doi. org/10.1016/j.chb.2017.12.015

Kaiser, G., Blömeke, S., Koenig, J., Busse, A., Doehrmann, M., \& Hoth, J. (2017). Professional competencies of (prospective) mathematics teachers-Cognitive versus situated approaches. Educational Studies in Mathematics, 94(2), 161-182. https://doi.org/10.1007/s10649-016-9713-8

Kaiser, G., Busse, A., Hoth, J., König, J., \& Blömeke, S. (2015). About the complexities of video-based assessments: Theoretical and methodological approaches to overcoming shortcomings of research on teachers' competence. International Journal of Science and Mathematics Education, 13(2), 369-387. https://doi. org/10.1007/s10763-015-9616-7

Kelcey, B., Hill, H. C., \& Chin, M. J. (2019). Teacher mathematical knowledge, instructional quality, and student outcomes: A multilevel quantile mediation analysis. School Effectiveness and School Improvement, 30(4), 398-431. https://doi.org/10.1080/09243453.2019.1570944

Kennedy, J. (2014). Characteristics of massive open online courses (moocs): A research review, $2009-2012$. Journal of Interactive Online Learning, 13(1), 1-16.

Kersting, N. B., Givvin, K. B., Thompson, B. J., Santagata, R., \& Stigler, J. W. (2012). Measuring usable knowledge: Teachers' analyses of mathematics classroom videos predict teaching quality and student learning. American Educational Research Journal, 49(3), 568-589. https://doi.org/10.3102/0002831212437853

Kesumawati, A., \& Utari, D. T. (2018, October). Predicting patterns of student graduation rates using Naïve Bayes classifier and support vector machine. In AIP conference proceedings (Vol. 2021, No. 1, p. 060005). AIP Publishing LLC.

Kilbertus, N., Ball, P. J., Kusner, M. J., Weller, A., \& Silva, R. (2020). The sensitivity of counterfactual fairness to unmeasured confounding. In Uncertainty in artificial intelligence (pp.616-626). Proceedings of Machine Learning Research (PMLR).

Kilgus, S. P., Chafouleas, S. M., Riley-Tillman, T. C., \& Welsh, M. E. (2012). Direct behavior rating scales as screeners: A preliminary investigation of diagnostic accuracy in elementary school. School Psychology Quarterly, 27(1), 41. https://doi.org/10.1037/a0027150

Klein, O., Hardwicke, T. E., Aust, F., Breuer, J., Danielsson, H., Hofelich Mohr, A., ljzerman, H., Nilsonne, G., Vanpaemel, W., \& Frank, M. C. (2018). A practical guide for transparency in psychological science. Collabra: Psychology, 4(1), 1-15.

Knievel, I., Lindmeier, A. M., \& Heinze, A. (2015). Beyond knowledge: Measuring primary teachers' subjectspecific competences in and for teaching mathematics with items based on video vignettes. International Journal of Science and Mathematics Education, 13(2), 309-329. https://doi.org/10.1007/s10763-014-9608-z

Koedinger, K., Cunningham, K., Skogsholm, A., \& Leber, B. (2008, June). An open repository and analysis tools for fine-grained, longitudinal learner data. In Educational data mining 2008.

Kosinski, M., Matz, S. C., Gosling, S. D., Popov, V., \& Stillwell, D. (2015). Facebook as a research tool for the social sciences: Opportunities, challenges, ethical considerations, and practical guidelines. American Psychologist, 70(6), 543. https://doi.org/10.1037/a0039210

Kosinski, M., Stillwell, D., \& Graepel, T. (2013). Private traits and attributes are predictable from digital records of human behavior. Proceedings of the National Academy of Sciences, 110(15), 5802-5805. https://doi. org/10.1073/pnas.1218772110 
Kotsiantis, S. B. (2012). Use of machine learning techniques for educational proposes: A decision support system for forecasting students' grades. Artificial Intelligence Review, 37(4), 331-344. https://doi.org/10.1007/s1046 2-011-9234-x

Kunter, M., Baumert, J., Blum, W., Klusmann, U., Krauss, S., \& Neubrand, M. (2013). Cognitive activation in the mathematics classroom and professional competence of teachers: Results from the COACTIV project. Springer Science \& Business Media.

Kunter, M., Klusmann, U., Baumert, J., Richter, D., Voss, T., \& Hachfeld, A. (2013). Professional competence of teachers: Effects on instructional quality and student development. Journal of Eduactional Psychology, 105, 805-820. https://doi.org/10.1037/a0032583

Kusner, M. J., \& Loftus, J. R. (2020). The long road to fairer algorithms. Nature Publishing Group.

Kusner, M. J., Loftus, J., Russell, C., \& Silva, R. (2017). Counterfactual fairness. In Proceedings of the 31st International Conference on Neural Information Processing Systems (pp. 4069-4079).

Lakens, D., Adolfi, F. G., Albers, C. J., Anvari, F., Apps, M. A. J., Argamon, S. E., Baguley, T., Becker, R. B., Benning, S. D., Bradford, D. E., Buchanan, E. M., Caldwell, A. R., Van Calster, B., Carlsson, R., Chen, S.-C., Chung, B., Colling, L. J., Collins, G. S., Crook, Z., ... Zwaan, R. A. (2018). Justify your alpha. Nature Human Behaviour, 2(3), 168-171. https://doi.org/10.1038/s41562-018-0311-x

Lambiotte, R., \& Kosinski, M. (2014). Tracking the digital footprints of personality. Proceedings of the IEEE, 102(12), 1934-1939. https://doi.org/10.1109/JPROC.2014.2359054

Lane, N. D., Miluzzo, E., Lu, H., Peebles, D., Choudhury, T., \& Campbell, A. T. (2010). A survey of mobile phone sensing. IEEE Communications Magazine, 48(9), 140-150. https://doi.org/10.1109/MCOM.2010.5560598

Laney, D. (2001). 3D data management: Controlling data volume, velocity and variety. META Group Research Note, $6,70$.

Lang, M., Binder, M., Richter, J., Schratz, P., Pfisterer, F., Coors, S., Au, Q., Casalicchio, G., Kotthoff, L., \& Bischl, B. (2019). mlr3: A modern object-oriented machine learning framework in R. Journal of Open Source Software, 4(44), 1903. https://doi.org/10.21105/joss.01903

Lindl, A., Krauss, S., Schilcher, A., \& Hilbert, S. (2020). Statistical methods in transdisciplinary educational research. Frontiers in Education, 5, 97. https://doi.org/10.3389/feduc.2020.00097

Lipowsky, F., Rakoczy, K., Pauli, C., Drollinger-Vetter, B., Klieme, E., \& Reusser, K. (2009). Quality of geometry instruction and its shortterm impact on students' understanding of the Pythagorean Theorem. Learning and Instruction, 19(6), 527-537. https://doi.org/10.1016/j.learninstruc.2008.11.001

Liu, B., Ding, M., Shaham, S., Rahayu, W., Farokhi, F., \& Lin, Z. (2021). When machine learning meets privacy: A survey and outlook. ACM Computing Surveys (CSUR), 54(2), 1-36. https://doi.org/10.1145/3436755

Liu, L. T., Dean, S., Rolf, E., Simchowitz, M., \& Hardt, M. (2018). Delayed impact of fair machine learning. arXiv preprint arXiv:1803.04383.

Lu, J., Liu, A., Dong, F., Gu, F., Gama, J., \& Zhang, G. (2018). Learning under concept drift: A review. IEEE Transactions on Knowledge and Data Engineering, 31(12), 2346-2363. https://doi.org/10.1109/TKDE.2018.2876857

Luo, G. (2016). A review of automatic selection methods for machine learning algorithms and hyper-parameter values. Network Modeling Analysis in Health Informatics and Bioinformatics, 5(1), 18. https://doi.org/10.1007/ s13721-016-0125-6

Lykourentzou, I., Giannoukos, I., Nikolopoulos, V., Mpardis, G., \& Loumos, V. (2009). Dropout prediction in elearning courses through the combination of machine learning techniques. Computers \& Education, 53(3), 950-965. https://doi.org/10.1016/j.compedu.2009.05.010

Markowetz, A., Blaszkiewicz, K., Montag, C., Switala, C., \& Schlaepfer, T. E. (2014). Psycho-informatics: Big data shaping modern psychometrics. Medical Hypotheses, 82(4), 405-411. https://doi.org/10.1016/j. mehy.2013.11.030

Marsh, H. W., Lüdtke, O., Nagengast, B., Trautwein, U., Morin, A. J., Abduljabbar, A. S., \& Köller, O. (2012). Classroom climate and contextual effects: Conceptual and methodological issues in the evaluation of grouplevel effects. Educational Psychologist, 47(2), 106-124. https://doi.org/10.1080/00461520.2012.670488

Mashek, D., \& Hammer, E. Y. (2011). Empirical research in teaching and learning: Contributions from social psychology (Vol. 4). John Wiley \& Sons.

Mashiloane, L., \& Mchunu, M. (2013). Mining for marks: A comparison of classification algorithms when predicting academic performance to identify “students at risk”. In R. Prasath, P. O’Reilly, \& T. Kathirvalavakumar (Eds.), Mining intelligence and knowledge exploration (pp. 541-552). Springer.

Maxwell, S. E. (2004). The persistence of underpowered studies in psychological research: Causes, consequences, and remedies. Psychological Methods, 9(2), 147. https://doi.org/10.1037/1082-989X.9.2.147

Molnar, C. (2020). Interpretable machine learning. Lulu.com.

Molnar, C., König, G., Herbinger, J., Freiesleben, T., Dandl, S., Scholbeck, C. A., Casalicchio, G., Grosse-Wentrup, M., \& Bischl, B. (2020). Pitfalls to avoid when interpreting machine learning models.

Montag, C., Błaszkiewicz, K., Sariyska, R., Lachmann, B., Andone, I., Trendafilov, B., Eibes, M., \& Markowetz, A. (2015). Smartphone usage in the 21st century: Who is active on WhatsApp? BMC Research Notes, 8(1), 331. https://doi.org/10.1186/s13104-015-1280-z 
Mullis, I. V., Martin, M. O., Foy, P., \& Hooper, M. (2017). Pirls 2016. International results in reading. International Association for the Evaluation of Educational Assessment (IEA).

Mullis, I. V., Martin, M. O., \& Loveless, T. (2016). 20 years of timss: International trends in mathematics and science achievement curriculum and instruction. TIMSS \& PIRLS International Study Center, Lynch School of Education, Boston College; International Association for the Evaluation of Educational Achievement (IEA).

Nitko, A. J. (1996). Educational assessment of students. ERIC.

OECD (2019a). Pisa 2018 results (volume i): What students know and can do. OECD Publishing.

OECD (2019b). Pisa 2018 results (volume ii): Where all students can succeed. OECD Publishing.

OECD (2019c). Pisa 2018 results (volume iii): What school life means for students' lives. OECD Publishing.

OECD (2019d). Talis 2018 results (volume i): Teachers and school leaders as lifelong learners. OECD Publishing.

OECD (2020a). Pisa 2018 results (volume iv): Are students smart about money?. OECD Publishing.

OECD (2020b). Talis 2018 results (volume ii): Teachers and school leaders as valued professionals. OECD Publishing.

O'Hara, R., \& Kotze, J. (2010). Do not log-transform count data. Methods in Ecology and Evolution, 1, 118-122.

Osborne, J. (2002). Notes on the use of data transformations. Practical Assessment, Research, and Evaluation, $8(1), 6$.

Osborne, J. (2010). Improving your data transformations: Applying the Box-Cox transformation. Practical Assessment, Research, and Evaluation, 15(1), 12.

Park, G., Schwartz, H. A., Eichstaedt, J. C., Kern, M. L., Kosinski, M., Stillwell, D. J., Ungar, L. H., \& Seligman, M. E. (2015). Automatic personality assessment through social media language. Journal of Personality and Social Psychology, 108(6), 934. https://doi.org/10.1037/pspp0000020

Porter, J. (2020). UK ditches exam results generated by biased algorithm after student protests. https://www. theverge.com/2020/8/17/21372045/uk-a-level-results-algorithmbiased-coronavirus-covid-19-pandemicuniversity-applications

Praetorius, A.-K., Klieme, E., Herbert, B., \& Pinger, P. (2018). Generic dimensions of teaching quality: The German framework of three basic dimensions. ZDM, 50(3), 407-426. https://doi.org/10.1007/s11858-018-0918-4

Ratner, B. (2017). Statistical and machine-learning data mining: Techniques for better predictive modeling and analysis of big data. CRC Press.

Ready, D. D., \& Wright, D. L. (2011). Accuracy and inaccuracy in teachers' perceptions of young children's cognitive abilities: The role of child background and classroom context. American Educational Research Journal, 48(2), 335-360. https://doi.org/10.3102/0002831210374874

Reilly, D., Neumann, D. L., \& Andrews, G. (2019). Gender differences in reading and writing achievement: Evidence from the national assessment of educational progress (NAEP). American Psychologist, 74(4), 445. https://doi.org/10.1037/amp0000356

Reis, H. T., \& Gable, S. L. (2000). Event-sampling methods. In H.T. Reis, \& C. Judd (Eds.), Handbook of research methods in social and personality psychology (p. 190-222). New York, NY:Cambridge University Press.

Rigaki, M., \& Garcia, S. (2020). A survey of privacy attacks in machine learning. arXiv preprint arXiv:2007.07646.

Ritzer, G., \& Stepnisky, J. (2020). Classical sociological theory. SAGE Publications, Incorporated.

Romero, C., \& Ventura, S. (2010). Educational data mining: A review of the state of the art. IEEE Transactions on Systems, Man, and Cybernetics, Part C (Applications and Reviews), 40(6), 601-618. https://doi.org/10.1109/ TSMCC.2010.2053532

Romero, C., \& Ventura, S. (2013). Data mining in education. Wiley Interdisciplinary Reviews: Data Mining and Knowledge Discovery, 3(1), 12-27. https://doi.org/10.1002/widm.1075

Romero, C., \& Ventura, S. (2020). Educational data mining and learning analytics: An updated survey. Wiley Interdisciplinary Reviews: Data Mining and Knowledge Discovery, 10(3), e1355.

Saeb, S., Lattie, E. G., Schueller, S. M., Kording, K. P., \& Mohr, D. C. (2016). The relationship between mobile phone location sensor data and depressive symptom severity. PeerJ, 4, e2537. https://doi.org/10.7717/peerj.2537

Saito, T., \& Watanobe, Y. (2020). Learning path recommendation system for programming education based on neural networks. International Journal of Distance Education Technologies (IJDET), 18(1), 36-64. https:// doi.org/10.4018/IJDET.2020010103

Samuel, A. L. (1959). Some studies in machine learning using the game of checkers. IBM Journal of Research and Development, 3(3), 210-229. https://doi.org/10.1147/rd.33.0210

Schmidt, W. H., Blömeke, S., Tatto, M. T., Hsieh, F.-J., Cogan, L., Houang, R. T., \& Schwille, J. (2011). Teacher education matters: A study of middle school mathematics teacher preparation in six countries. Teachers College Press, Columbia University New York.

Schoedel, R., Au, Q., Völkel, S. T., Lehmann, F., Becker, D., Bühner, M., Bischl, B., Hussmann, H., \& Stachl, C. (2018). Digital footprints of sensation seeking. Zeitschrift Für Psychologie, 226(4), 232-245. https://doi. org/10.1027/2151-2604/a000342

Schoedel, R., Pargent, F., Au, Q., Völkel, S. T., Schuwerk, T., Bühner, M., \& Stachl, C. (2020). To challenge the morning lark and the night owl: Using smartphone sensing data to investigate day-night behaviour patterns (J. Rauthmann, Ed.). European Journal of Personality, 34(5), 733-752. https://doi.org/10.1002/per.2258 
Schoenfeld, A. H. (2018). Research commentary: On replications. Journal for Research in Mathematics Education, 49(1), 91-97. https://doi.org/10.5951/jresematheduc.49.1.0091

Schölkopf, B., \& Smola, A. J. (2003). A short introduction to learning with kernels. In Advanced lectures on machine learning (pp. 41-64). Springer.

Schubert, F., \& Becker, R. (2010). Social inequality of reading literacy: A longitudinal analysis with cross-sectional data of pirls 2001 and pisa 2000 utilizing the pair wise matching procedure. Research in Social Stratification and Mobility, 28(1), 109-133. https://doi.org/10.1016/j.rssm.2009.12.007

Sculley, D., Holt, G., Golovin, D., Davydov, E., Phillips, T., Ebner, D., Chaudhary, V., \& Young, M. (2014). Machine learning: The high interest credit card of technical debt.

Servia-Rodríguez, S., Rachuri, K. K., Mascolo, C., Rentfrow, P. J., Lathia, N., \& Sandstrom, G. M. (2017). Mobile sensing at the service of mental well-being: A large-scale longitudinal study. In 26th international world wide web conference, www 2017, International World Wide Web Conferences Steering Committee.

Shearer, C. (2000). The CRISP-DM model: The new blueprint for data mining. Journal of Data Warehousing, 5(4), 13-22.

Shmueli, G. (2010). To explain or to predict? Statistical Science, 25(3), 289-310. https://doi.org/10.1214/10-STS330

Shulman, L. S. (1986). Those who understand: Knowledge growth in teaching. Educational Researcher, 15(2), 4-14. https://doi.org/10.3102/0013189X015002004

Simmons, J. P., Nelson, L. D., \& Simonsohn, U. (2011). False-positive psychology: Undisclosed flexibility in data collection and analysis allows presenting anything as significant. Psychological Science, 22(11), 1359-1366. https://doi.org/10.1177/0956797611417632

Simon, R. (2007). Resampling strategies for model assessment and selection. In W. Dubitzky, M. Granzow, \& D. Berrar (Eds.), Fundamentals of data mining in genomics and proteomics (pp. 173-186). Springer US.

Solaiman, I., Brundage, M., Clark, J., Askell, A., Herbert-Voss, A., Wu, J., Radford, A., \& Wang, J. (2019). Release strategies and the social impacts of language models. arXiv 1908.09203. http://arxiv.org/ abs/1908.09203

Stachl, C., Au, Q., Schoedel, R., Gosling, S. D., Harari, G. M., Buschek, D., Theres, S., Olkel, V., Schuwerk, T., Oldemeier, M., Ullmann, T., Hussmann, H., Bischl, B., \& Bühner, M. (2020). Predicting personality from patterns of behavior collected with smartphones. Proceedings of the National Academy of Sciences, 117(30), 17680-17687. https://doi.org/10.1073/pnas.1920484117

Stachl, C., Hilbert, S., Au, J.-Q., Buschek, D., De Luca, A., Bischl, B., Hussmann, H., \& Bühner, M. (2017). Personality traits predict smartphone usage. European Journal of Personality, 31(6), 701-722. https://doi. org/10.1002/per.2113

Stachl, C., Pargent, F., Hilbert, S., Harari, G. M., Schoedel, R., Vaid, S., Gosling, S. D., \& Bühner, M. (2020). Personality research and assessment in the era of machine learning. European Journal of Personality, 34(5), 613-631. https://doi.org/10.1002/per.2257

Strauss, V. (2015). Master teacher suing New York state over 'ineffective' rating is going to court. https://www. washingtonpost.com/news/answer-sheet/wp/2015/08/09/master-teacher-suing-new-york-stateover-ineff ective-rating-is-going-to-court/

Strobl, C., Boulesteix, A.-L., Kneib, T., Augustin, T., \& Zeileis, A. (2008). Conditional variable importance for random forests. BMC Bioinformatics, 9(1), 307. https://doi.org/10.1186/1471-2105-9-307

Tanuwidjaja, H. C., Choi, R., Baek, S., \& Kim, K. (2020). Privacy-preserving deep learning on machine learning as a service-A comprehensive survey. IEEE Access, 8, 167425-167447. https://doi.org/10.1109/ ACCESS.2020.3023084

Tay, L., Woo, S. E., Hickman, L., \& Saef, R. M. (2020). Psychometric and validity issues in machine learning approaches to personality assessment: A focus on social media text mining. European Journal of Personality, 34(5), 826-844. https://doi.org/10.1002/per.2290

Teasley, S. D. (2019). Learning analytics: Where information science and the learning sciences meet. Information and Learning Sciences, 120(1/2), 59-73. https://doi.org/10.1108/ILS-06-2018-0045

Theobald, E. J., Aikens, M., Eddy, S., \& Jordt, H. (2019). Beyond linear regression: A reference for analyzing common data types in discipline based education research. Physical Review Physics Education Research, 15(2), 020110. https://doi.org/10.1103/PhysRevPhysEducRes.15.020110

Tibshirani, R. (1996). Regression shrinkage and selection via the lasso. Journal of the Royal Statistical Society: Series B (Methodological), 58(1), 267-288.

Tohidi, H., \& Jabbari, M. M. (2012). The effects of motivation in education. Procedia-Social and Behavioral Sciences, 31, 820-824. https://doi.org/10.1016/j.sbspro.2011.12.148

Turabik, T., \& Baskan, G. A. (2015). The importance of motivation theories in terms of education systems. Procedia-Social and Behavioral Sciences, 186, 1055-1063. https://doi.org/10.1016/j.sbspro.2015.04.006

Wachter, S., Mittelstadt, B. D., \& Russell, C. (2017). Counterfactual explanations without opening the black box: Automated decisions and the GDPR. CoRR, abs/1711.00399. arXiv 1711.00399. http://arxiv.org/ abs/1711.00399

Waljee, A. K., Higgins, P. D., \& Singal, A. G. (2014). A primer on predictive models. Clinical and Translational Gastroenterology, 5(1), e44. https://doi.org/10.1038/ctg.2013.19 
Ward, J. S., \& Barker, A. (2013). Undefined by data: A survey of big data definitions. arXiv preprint arXiv:1309.5821. Weinert, F. E. (2001). Concept of competence: A conceptual clarification.

Wisniewski, B., Zierer, K., Dresel, M., \& Daumiller, M. (2020). Obtaining secondary students' perceptions of instructional quality: Two-level structure and measurement invariance. Learning and Instruction, 66, 101303. https://doi.org/10.1016/j.learninstruc.2020.101303

Wulf, C. (2003). Educational science. Hermeneutics, empirical research, critical theory. Waxmann.

Yarkoni, T., \& Westfall, J. (2017). Choosing prediction over explanation in psychology: Lessons from machine learning. Perspectives on Psychological Science, 12(6), 1100-1122. https://doi.org/10.1177/1745691617 693393

Ylijoki, O., \& Porras, J. (2016). Perspectives to definition of big data: A mapping study and discussion. Journal of Innovation Management, 4(1), 69-91. https://doi.org/10.24840/2183-0606_004.001_0006

Yoo, J. E. (2018). Timss 2011 student and teacher predictors for mathematics achievement explored and identified via elastic net. Frontiers in Psychology, 9, 317. https://doi.org/10.3389/fpsyg.2018.00317

Youyou, W., Kosinski, M., \& Stillwell, D. (2015). Computer-based personality judgments are more accurate than those made by humans. Proceedings of the National Academy of Sciences, 112(4), 1036-1040. https://doi. org/10.1073/pnas.1418680112

Zhao, Q., \& Hastie, T. (2019). Causal interpretations of black-box models. Journal of Business \& Economic Statistics, 39(1), 272-281. https://doi.org/10.1080/07350015.2019.1624293

Zou, H., \& Hastie, T. (2005). Regularization and variable selection via the elastic net. Journal of the Royal Statistical Society: Series B (Statistical Methodology), 67(2), 301-320. https://doi.org/10.1111/j.1467-9868.2005.00503.x

\section{SUPPORTING INFORMATION}

Additional supporting information may be found in the online version of the article at the publisher's website.

How to cite this article: Hilbert, S., Coors, S., Kraus, E., Bischl, B., Lindl, A., Frei, M., Wild, J., Krauss, S., Goretzko, D., \& Stachl, C. (2021). Machine learning for the educational sciences. Review of Education, 9, e3310. https://doi.org/10.1002/ $\underline{\text { rev3.3310 }}$ 